\title{
Magnetic flux closure in mutant magnetotactic bacteria elucidates a key signature of magnetofossils
}

\section{Matthieu Amor}

Aix-Marseilles University/CNRS https://orcid.org/0000-0002-4299-1493

\section{Juan Wan}

University of California, Berkeley

\section{Ramon Egli}

Central Institute for Meteorology and Geodynamics https://orcid.org/0000-0001-6825-4742

Julie Carlut

Université de Paris

Christiphe Gatel

CEMES

\section{Ingrid Marie Andersen}

Centre d'Élaboration de Matériaux et d'Etudes Structurales

\section{Etienne Snoeck}

Centre d'Élaboration de Matériaux et d'Etudes Structurales

Arash Komeili ( $\sim$ komeili@berkeley.edu )

University of California, Berkeley

\section{Article}

Keywords: Biomineralization, Magnetofossils, Vortex State, First-Order Reversal Curves, Electron Holography

Posted Date: February 12th, 2021

DOl: https://doi.org/10.21203/rs.3.rs-187824/v1

License: (c) (i) This work is licensed under a Creative Commons Attribution 4.0 International License. Read Full License 
3 Matthieu Amor ${ }^{a, b^{*}}$, Juan Wan ${ }^{*}$, Ramon Eglic ${ }^{{ }^{d s}}$, Julie Carlut ${ }^{\mathrm{d}}$, Christophe Gatel, ${ }^{\mathrm{cef}}$ Ingrid Marie

7 'Aix-Marseille University, CNRS, CEA, UMR7265 Biosciences and Biotechnology

8 Institute of Aix-Marseille, CEA Cadarache, Saint-Paul-lez-Durance, F-13108, France

9 'Zentralanstalt für Meteorologie und Geoynamik (ZAMG), Hohe Warte 38, A-1190

11 Université de Paris, Institut de Physique du Globe de Paris, CNRS, 75005 Paris, France

20 Keywords: Biomineralization, Magnetofossils, Vortex State, First-Order Reversal

21 Curves, Electron Holography
Vienna, Austria

${ }^{\text {e }}$ CEMES CNRS, 29 rue Jeanne Marvig, F-31055 Toulouse, France

‘Université de Toulouse, F-31077 Toulouse, France

:Department of Molecular and Cell Biology, University of California, Berkeley, CA

94720-3200

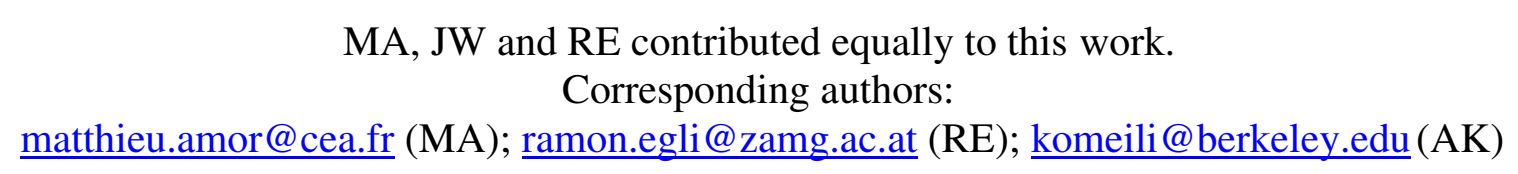

MA, JW and RE contributed equally to this work.

Corresponding authors: 


\section{Abstract}

23 Magnetotactic bacteria (MTB) produce single- or multi-stranded chains of magnetic

24 nanoparticles that contribute to the magnetization of sedimentary rocks. Their magnetic

25 fingerprint can be detected in ancient geological samples, and serve as a unique

26 biosignature of microbial life. However, fossilized assemblages bear contradictory

27 signatures pointing to magnetic components that have distinct origin(s). Here, we produce

28 mutant bacteria to mimic MTB producing multi-stranded chains that cannot be cultivated

29 in the laboratory, and show that the unresolved magnetic signatures are fully compatible

30 with the contribution of MTB synthesizing multi-stranded nanoparticle chains and with

31 fold-collapsed single-stranded chains. These structures generate magnetic flux-closing

32 configurations while maintaining high remanent magnetizations. This work has important

33 paleoclimatic, paleontological and phylogenetic implications, as it provides a novel tool to

34 differentiate distinct MTB lineages (single- vs multi-stranded nanoparticle chains) which

35 will enable the tracking of the evolution of some of the most ancient biomineralizing

36 organisms in a time-resolved manner.

37

38 


\section{Introduction}

40 Magnetite $\left(\mathrm{Fe}_{3} \mathrm{O}_{4}\right)$ is a ubiquitous iron oxide found in sediments and in many sedi-

41 mentary rocks. It is a major carrier of sediment and rock magnetization used for numerous

42 applications in Earth sciences including paleomagnetic, paleoclimate, paleoenvironmental,

43 paleogeographic and paleontological reconstructions '. In addition to lithogenic crystals,

44 secondary magnetite particles form in sediments through chemical and diagenetic

45 processes, but can also be produced by bacteria ${ }^{2}$.

46 Magnetotactic bacteria (MTB) are the only known microorganisms producing

47 intracellular nanoparticles of magnetite in organelles called magnetosomes under a

48 genetically controlled pathway ${ }^{3}$. They represent some of the most ancient biomineralizing

49 organisms, with a proposed origin of $\sim 3 \mathrm{Ga}^{4}$. They are markers of oxic-anoxic transition

50 zones in aquatic and sedimentary environments ${ }^{5.6}$, making them useful paleoenvironmental

51 indicators ${ }^{7-9}$. Magnetosomes are assembled in chains, and provide the bacteria with a

52 magnetic dipole for navigation purposes ${ }^{10}$. When MTB cells die, their magnetite chains can

53 be trapped into sediments and subsequently fossilized ${ }^{11,12}$. MTB fossils (hereafter referred

54 to as magnetofossils) can be preserved over geological times and contribute to sedimentary

55 paleomagnetic and environmental records ${ }^{13,14}$. Their unambiguous identification, and

56 discrimination from abiotic magnetite, would thus provide strong constraints on the

57 evolution of life, biomineralization processes, and environmental conditions over

58 geological times.

59 First-order reversal curve (FORC) diagrams, which rely on the measurement of partial

60 hysteresis curves, can be used to discriminate isolated single-domain (SD) magnetite

61 particles and magnetofossils from other magnetic structures, such as SD particle clusters 
62 and larger lithogenic (titano)magnetite crystals containing magnetic vortices (VO) ormul-

63 tiple magnetic domains (MD) ${ }^{\text {s.1.6, }}$, through a sharp signature called the central ridge ${ }^{1.1 .18}$, down

64 to concentrations inaccessible with other characterization methods. Principal component

65 analyses (PCA) of FORC diagrams obtained from magnetofossil-rich sediments typically

66 yield mixtures of MD and VO signatures with a large vertical dispersion around the SD-

67 related central ridge that contribute to $40-65 \%$ of the total magnetization (Table 1) ${ }^{19}$. These

68 results can be explained by mixtures of (i) well-dispersed non-interacting SD particles or

69 linear chains of such particles contributing to the central ridge, and (ii) clumped SD and/or

70 VO particles contributing to the remaining parts of the FORC distribution. The associated

71 magnetic hysteresis parameters (see glossary in the SI appendix) describing the shape of

72 the hysteresis loop are close to the theoretical limit for non-interacting SD particles and

73 magnetosome chains (Table 1). In particular, the hysteresis squareness (the ratio of

74 remanent and saturation magnetizations) of $\sim 0.45$ is unrealistically large for samples

75 containing strongly interacting SD particles or VO particles associable with the observed

76 FORC contributions around the central ridge ${ }^{2022}$ (Fig. 1). Collapsed obtained from MTB

77 cultures after cell lysis are instead characterized by an excessively low hysteresis

78 squareness ${ }^{2324}$ (Table 1, Fig. 1). Thus, current interpretations of these magnetic assemblages

79 appear to miss structures possessing the high hysteresis squareness typical of SD particles,

80 as well as a mixed FORC signature with a central ridge and VO contributions.

81 Possible candidates for such structures include multiple strands of magnetosomes

82 produced by certain $\mathrm{MTB}^{25}$, and specific, mechanically meaningful forms of chain collapse,

83 such as double strands resulting from bending single-stranded chains beyond the elastic

84 limit ${ }^{26}$. Single- and multi-stranded chains in MTB are in a native SD state that maximizes 
85 the total magnetic moment of the cell, with the magnetic moments of all magnetosomes

86 being parallel to the chain axis ${ }^{27}$. This is the only possible magnetic configuration for

87 single-stranded chains, but not for multiple strands, in which intermediate states with lower

88 magnetic moments can be nucleated with the application of strong external fields ${ }^{27}$.

89 Intermediate states are formed when the magnetic moment of one strand is reversed with

90 respect to the others, or when the magnetic moments of individual particles form complex

91 patterns ${ }^{28}$. The common characteristic of these so-called flux-closure (FC) configurations

92 is the formation of closed field line loops inside the chain body, which suppress the external

93 stray field of SD states. Transitions between SD and FC states, and vice-versa, generate

94 FORC diagram contributions outside the region occupied by the central ridge, resembling

95 those of $\mathrm{VO}{ }^{15,16}$ or strongly interacting SD particles ${ }^{29}$. Unlike these latter systems with a

96 small hysteresis squareness, native multi-stranded chains are characterized by squareness

97 values close to 0.5 due to the stability of SD states, including the native one, in zero or very

98 small ambient fields ${ }^{30}$.

99 Here, we propose that the unresolved magnetic signature of magnetofossils can be

100 explained by combinations of intact or elastically deformed single- or multi-stranded

101 magnetosome chains (non-collapsed), and fold-collapsed chains. Nucleation and annihi-

102 lation of FC magnetic states explains the VO signatures in FORC diagrams of magneto-

103 fossil-bearing sediments, while the strong uniaxial anisotropy of intact and collapsed

104 chains ensures that they still possess SD-like remanence and SD-like hysteresis squareness

105 of $\sim 0.5$. Our claim is supported by micromagnetic simulations of single- and double-

106 stranded chains made of equant and prismatic magnetosomes. These simulations should be

107 validated using MTB with multi-stranded chains. However, no such MTB are available for 
108 cultures in the laboratory. To overcome this limitation, we produced a mutant of the

109 magnetotactic bacterium Magnetospirillum magneticum strain AMB-1 (AMB-1)

110 synthesizing folded magnetite chains, whose looped structure is topologically identical to

111 double-stranded chain configurations in wild-type uncultured MTB. From magnetic

112 measurements and off-axis electron holography characterizations, we demonstrate that the

113 folded chains produced by the AMB-1 mutant generate FC magnetic signatures similar to

114 those obtained with our micromagnetic simulations, while lacking the hysteresis

115 squareness of the simulations and of natural magnetofossil signatures. This demonstrates

116 that the unique magnetic fingerprints of magnetofossils originate from the magnetosome

117 arrangements with strong uniaxial anisotropy, distinct from that of clusters and looped

118 chains. This work resolves longstanding and ongoing controversies about the interpretation

119 of sedimentary sources of SD magnetite, establishing a theoretical and experimental

120 framework that explains the magnetofossil signature and provides insights into the

121 diversity of ancient MTB populations.

122

\section{Results}

124 Micromagnetic modeling. To determine whether native multi-stranded and fold-

125 collapsed magnetosome chains could explain the combination of SD and VO properties

126 with SD hysteresis squareness typical of MTB, we first modeled their FORC distributions.

127 Micromagnetic modeling of high-resolution FORC measurements was performed for

128 selected magnetosome morphologies and chain configurations representative of intact or

129 elastically deformed chains, and of fold-collapsed chains. The magnetic properties of the

130 simulated structures are determined mainly by two factors: the anisotropy of individual 
131 magnetosomes which is controlled by their shape and crystal orientation, and magnetostatic

132 interactions. Magnetosome shapes can be classified as equant (cuboctahedral, octahedral),

133 elongated (prismatic, elongated octahedral), and highly elongated (tooth- and bullet-

134 shaped) ${ }^{25}$. These categories form well-defined clusters of crystal sizes within the SD

135 stability range ${ }^{31,2}$ (Fig. S1).

136 The geometry of intact single-stranded chains is controlled by the gap between magneto-

137 somes, the decrease of immature magnetosomes sizes towards the chain ends (tapering),

138 and elastic bending. Magnetosomes in multi-stranded chains are staggered: each

139 magnetosome in one strand faces the gap between two magnetosomes of a nearby strand

140 (Fig. S2). This arrangement favors the formation of strand bundles with a consistent

141 magnetic polarity and minimizes repulsive forces ${ }^{27}$. Contrastingly, magnetosomes in fold-

142 collapsed chains are arranged side-to-side, and size tapering occurs only at one end, the

143 other end being the kink point of the native chain. The side-to-side magnetosome

144 arrangement maximizes lateral attractive forces ${ }^{28}$.

145 The full variability of natural chains can be only partially reproduced, because most

146 geometric parameters must be extrapolated from few observations. Therefore, we selected

147 combinations of two magnetosome morphologies (cuboctahedral and prismatic), and three

148 chain geometries (single-stranded, native double-stranded, and double-stranded from

149 collapse by folding), as representative examples of magnetic endmembers dictated by the

150 magnetosome aspect ratio (equant $v s$. elongated), and the existence of flux-closure

151 configurations (single- $v s$. double-stranded). FORC measurements were simulated after

152 generating 40,000 synthetic chains with random orientations and realistic geometries for 
153 each of the six categories obtained by combining the above-mentioned magnetosome

154 morphologies and chain configurations (Figs. S3-S6, for double-stranded chain examples).

155 The simulated FORC diagrams of single-stranded chains (Fig. 2A and 2D) possess all

156 the expected characteristics that have been predicted theoretically ${ }^{33}$ and verified on cultured

157 MTB ${ }^{24}$. The corresponding coercivity distributions are slightly narrower and biased

158 towards $\sim 30 \%$ higher fields with respect to the so-called biogenic soft (BS) and hard (BH)

159 components commonly found in magnetofossil-rich sediments and attributed to intact

160 chains of equidimensional and elongated magnetosomes, respectively ${ }^{193435}$. Simulated

161 FORC diagrams of double-stranded chain configurations feature flux-closure signatures

162 (Fig. 2B and 2E). These features are fully developed only in fold-collapsed chains of equant

163 magnetosomes, as seen by the constricted hysteresis loop (Fig. S7) and the clearly

164 identifiable positive and negative doublets above and below the central ridge (Fig. 2C).

165 However, contrary to the AMB-1 mutant (see below) and to existing examples of magnetic

166 systems featuring this type of FORC signature ${ }^{152236}$, the hysteresis squareness is only slightly

167 lowered with respect to the SD case $\left(M_{\mathrm{n}} / M_{\mathrm{s}}=0.471\right.$, with $M_{\mathrm{s}}$ and $M_{\mathrm{s}}$ corresponding to the

168 remanent and saturation magnetizations, respectively) (Table 1, Fig. 1), and fully

169 compatible with magnetofossil signatures ${ }^{20}$. Squareness values close to 0.5 for all simulated

170 double-stranded chains means that they possess stable SD states in zero field, as observed

171 on native chains ${ }^{3}$, and unlike the topologically equivalent structures of the AMB-1 mutant.

172 The SD stability of multi-stranded chains originates from their strong uniaxial anisotropy,

173 which is not shared by other natural structures with FC states. Uniaxial anisotropy is further

174 enhanced by magnetosome elongation and by the staggered crystal arrangement. 
175 The central ridge coercivity distributions of double-stranded chains are remarkably

176 similar to those of single-stranded chains made of the same type of magnetosomes, there-

177 fore contributing in a similar manner to the so-called BS and BH coercivity components of

178 magnetofossil signatures. The other coercivity distributions, on the other hand, contain

179 additional low-field contributions related to FC nucleation and annihilation. In the case of

180 equant magnetosomes, these contributions produce a second peak (fold-collapsed) or a

181 shoulder (native) around $15 \mathrm{mT}$. In the case of prismatic magnetosomes, distributions are

182 wider, and the peak position is lowered by $\sim 10 \mathrm{mT}$ with respect to the central ridge.

183 Our model thus reproduces well the unresolved magnetic signatures observed in

184 sedimentary rocks. However, the unique association of this signature with single- and

185 multi-stranded chains, which does not require previously proposed forms of chain collapse

186 or alteration [e.g. clumping ${ }^{23}$ and folding ${ }^{s 3}$, needs to be experimentally demonstrated.

187

188 Mutant AMB-1 strain with looped chains. Given that no MTB with multi-stranded

189 chains are available in culture for laboratory experiments, we generated a mutant AMB-1

190 strain making looped magnetosome chains (Fig. 3). Such mutant can serve as a useful term

191 of comparison given by chain configuration with FC states. Magnetosome formation in

192 MTB requires genes contained in a specific portion of the genome called the magnetosome

193 island (MAI) ${ }^{39}$. AMB-1 genome also contains a small version of the magnetosome island

194 called the magnetotaxis islet (MIS) ${ }^{\star 0}$. The MIS contains several genes, some of which

195 perform redundant functions with their homologs in the MAI "4. Previous work in

196 Magnetospirillum gryphiswaldense strain MSR-1 (MSR-1), a close relative of AMB-1,

197 showed that the loss of the MAI gene mamJ results in collapse and aggregation of 
198 magnetosome chains ${ }^{42}$. Surprisingly, the deletion of mamJ and its other MAI homolog limJ

199 in AMB-1 does not produce collapsed chains and instead results in minor disruptions to

200 the continuity of the chain ${ }^{43}$. We reasoned that redundant functions within the MIS might

201 account for the dramatic differences between mamJ mutants in AMB-1 and MSR-1.Thus,

202 a mutant strain ( $\Delta$ mamJ $\Delta$ limJ $\Delta$ MIS) was produced by deleting the entire MIS and two MAI

203 genes (mamJ and $\lim J$ ) from AMB-1 genome. Unlike the mamJ MSR-1 mutant, which

204 produces agglomerated magnetosomes, the phenotype of the AMB-1 mutant contains

205 looped magnetosome chains with necklace structures located either at one of the poles or

206 at the center of the cell (Fig. 3). Magnetite in the mutant strain falls in the stable SD size

207 range ${ }^{31}$. The magnetic behavior of bacteria was assessed using the magnetic coefficient

$208\left(C_{\mathrm{ms}}\right)$, which relies on the differential measurement of a culture's optical density when a 209 magnet is oriented either vertically or horizontally close to the cell suspension. $C_{\operatorname{mg}}$ is

210 defined as the ratio of the maximum and minimum optical density measured, and quantifies

211 the capacity of bacteria to orientate along an external magnetic field. $C_{\mathrm{mg}}$ values at the end

212 of bacterial growth were $1.82 \pm 0.09$ and $1.09 \pm 0.01$ for wild-type and mutant AMB-1,

213 respectively, demonstrating a very limited orientation capability for the mutant strain.

214 Native magnetic states of wild-type and mutant AMB-1 cells. The low $\mathrm{C}_{\operatorname{mg}}$ values in

215 the mutant AMB-1 strain suggests that its looped magnetosome chain configurations

216 possess a low net magnetic moment. We thus investigated the magnetic state of wild-type

217 and mutant AMB-1 strains at the population level by comparing the magnetization of two

218 different preparations of aqueous cell suspensions in a maximum external field of $2 \mathrm{mT}$,

219 using a vibrating-sample magnetometer (VSM). The external field is sufficiently large to

220 partially align the cells, but not large enough to alter their native magnetic configuration. 
221 The first preparation contains cells directly taken from the culture: in this case, the

222 suspension magnetization is expected to be proportional to the mean strength of the native

223 magnetic moments. In the second preparation, the suspension has been previously exposed

224 to a $200 \mathrm{mT}$ field. This field is sufficiently strong to reset the native magnetic states,

225 replacing them with a saturation remanent state carrying the maximum magnetic moment

226 that can be maintained in a null field.

227 Wild-type cells containing straight magnetite chains already possess a saturation rema228 nent state. Exposing them to a large field will thus not change the magnetization of the

229 suspension. In practice, a $\sim 40 \%$ increase is observed after applying a $200 \mathrm{mT}$ field (Fig.

$2304 \mathrm{~A})$. This increase might be explained by the growth of aligned chain fragments with 231 opposite native polarities within the same cell ${ }^{4}$. In this case, the application of a strong 232 external field imparts the same polarity to all fragments, increasing the cell's total magnetic 233 moment. A drastically different behavior is observed with the AMB-1 mutant: an 18-fold 234 magnetization increase is obtained after applying a $200 \mathrm{mT}$ field (Fig. 4D). Along with the 235 magnetic behavior assays $\left(C_{\mathrm{ms}}\right)$, this result indicates a vanishingly small native magnetic 236 moment of the AMB-1 mutant, which is compatible with a FC configuration of its looped 237 chains. The sigmoidal shape of the magnetization curve of the $200 \mathrm{mT}$-treated suspension 238 in fields up to $2 \mathrm{mT}$ can be explained by the concurring effects of cell alignment, which is 239 responsible for the curve slope above $0.5 \mathrm{mT}$, and the additional magnetization induced by 240 the reversible rotation of the magnetic moments of individual crystals towards the external 241 field. The induced magnetization is larger for high-energy magnetic configurations, such 242 as those created in the AMB-1 mutant by the application of a strong field, than for low- 
243 energy magnetic configurations, such as the native SD state of wild-type cells and the 244 native FC state of the mutant.

245 Electron holography. VSM characterizations of mutant AMB-1 demonstrated a

246 vanishingly small magnetic moment of the bacteria which may be related to the looped

247 chain structure. To further confirm this hypothesis, we mapped the magnetic flux in the

248 fold-collapsed magnetosome chains at the single-crystal level with off-axis electron

249 holography. Electron holography is an interferometric method that can correlate

250 morphological and local magnetic characterizations of magnetic materials. It allows

251 quantitative mapping of the in-plane flux inside the magnetite chains produced by AMB-1

252 at the nanometer scale. Mapping of the magnetic flux in magnetosome chains produced in 253 wild-type AMB-1 (Figs. 4B and 4C) indicates that the magnetic moments of individual 254 magnetosomes are nearly parallel to the chain axis, as previously observed ${ }^{45}$. In contrast, 255 necklace structures of the mutant strain display a closed magnetic flux (Figs. 4E and 4F). 256 This configuration has a zero-net magnetic moment, up to small fluctuations due to 257 asymmetries (e.g. larger crystals on one side of the structure), confirming the origin of the 258 vanishing native magnetic moments of the AMB-1 mutant deduced from magnetic 259 measurements.

260 FORC measurements. The analysis of the mutant AMB-1 shows that its looped chains 261 can be a reasonable mimic for naturally occurring multi-stranded chains as well as fold262 collapsed magnetosome chains. Thus, it was selected to test the hypothesis that multi263 stranded chain configurations can generate FORC signatures similar to the unresolved ones 264 observed in sediments. The magnetic properties of the wild-type and mutant AMB-1 strains 265 were characterized with high-resolution FORC analyses using a VSM. Wild-type AMB-1 
266 (Fig. 5A) shows the central ridge typical of isolated uniaxial SD particles and magnetosome

267 chains, as well as a negative-positive doublet in the lower quadrant, nearly antisymmetric

268 about the $B_{a}=-B_{c}$ diagonal, produced by the reversible rotation of magnetic moments in

269 the applied field ". The magnetically viscous contribution of immature chains is

270 recognizable from the presence of a vertical ridge along $B_{\mathrm{c}}=0^{46}$, and from the central ridge

271 extending down to $B_{c}=0$. These chains are made of magnetosomes with sizes close to the

272 lower SD stability limit (between 12 and $17 \mathrm{~nm}$ for equant crystals) (Fig. 3) ${ }^{32}$. The

273 hysteresis squareness $\left(M_{s} / M_{s}=0.475\right)$ is slightly smaller than the ideal value for single-

274 stranded chains, probably because of a small superparamagnetic contribution associated

275 with isolated immature crystals as confirmed by electron microscopy (Fig. 3). The

276 coercivity distributions obtained from FORC data subsets possess similar, unimodal

277 characteristics with a peak at $28-30 \mathrm{mT}$ and the right tail extending to a maximum of $60-$ $27870 \mathrm{mT}$.

279 The mutant AMB-1 strain shares these features (i.e. central ridge, viscous component 280 and rotations of magnetic moments in the applied field), albeit over different field ranges, 281 but also exhibits additional contributions (Fig. 5B), consisting of a doublet of positive lobes 282 at $B_{\mathrm{c}} \approx 12 \mathrm{mT}$ and $B_{\mathrm{a}} \approx \pm 8 \mathrm{mT}$, and a doublet of negative lobes at $B_{\mathrm{c}} \approx 25 \mathrm{mT}$ and $B_{\mathrm{a}} \approx \pm 3$ $283 \mathrm{mT}$, almost symmetrically arranged above and below the central ridge. These lobes are the 284 typical hallmark of FC nucleation and annihilation "s, as seen with VO particles ${ }^{22}$ and small 285 magnetosome clusters produced by the $\Delta m a m J$ mutant of MSR-1 ${ }^{36}$. The hysteresis loop is 286 slightly constricted (Fig. S8) with a much smaller squareness (Table1, Fig. 1), which is 287 caused by necklace-like magnetosome arrangements that do not possess stable SD-like 288 magnetic states in a null field due to insufficient or absent uniaxial anisotropy. Constricted 
289 hysteresis loops are typically the result of bimodal coercivity distributions, which, in this 290 case, consist of a main peak at $\sim 2 \mathrm{mT}$, and a shoulder at $\sim 25 \mathrm{mT}$ (Fig. 5D). The main peak 291 of the central ridge, close to the origin, is caused by thermally activated transitions between 292 SD states of structures made of immature magnetosomes, including isolated crystals. FC 293 nucleation from the negative SD state (feature 4 in Fig. 5B) contributes additionally to the 294 main peak of the other two coercivity distributions, explaining its larger amplitude with 295 respect to the central ridge. A single mechanism, namely the annihilation of FC states 296 around $+25 \mathrm{mT}$, explains the existence of a shoulder with similar amplitude in all three 297 coercivity distributions.

298 The lack of low-coercivity central ridge contributions from FC nucleation is acommon 299 feature of all magnetic systems with coexisting SD-like and FC states and is a key point 300 for understanding magnetofossil signatures. Another key point is the lowered hysteresis 301 squareness of the AMB-1 mutant. Its incompatibility with the magnetic signature of 302 magnetofossil-rich sediments permits the exclusion of looped magnetosome 303 configurations, such as those produced by laboratory-induced chain collapse ${ }^{\text {" }}$ and by the 304 self-avoiding random walk algorithm used to simulate magnetofossil chains with various 305 collapse degrees ${ }^{38.48}$.

306 In summary, the combination of magnetic modelling and measurements demonstrate 307 that magnetic assemblages with coexisting central ridge, SD-like and VO (features 4, 5 and 3086 in Fig. 5B) states can be explained by the presence of multi-stranded magnetosome chains 309 rather than multiple components with distinct origins and magnetic states. The

310 conservation of high hysteresis squareness typical of intact magnetosome chains points to 
311 native multi-stranded chains produced by specific MTB lineages, while lower hysteresis

312 squareness being incompatible with aligned magnetosome particles also indicates the

313 presence of fold-collapsed chains.

\section{Discussion}

315 Magnetic assemblages showing concurrent fingerprints of central ridges (no

316 magnetostatic interactions) and vertical scattering of the FORC distribution (large

317 magnetostatic interactions) were previously interpreted as mixings of several magnetite

318 sources including magnetofossils, magnetosome clusters, MD and VO particles ${ }^{1949}$. These

319 sources have various origins and provide insights into the geobiological history of the

320 sediments hosting the magnetic assemblages. However, mixings of the above-mentioned

321 magnetic sources cannot provide a satisfying explanation for the high hysteresis squareness

322 typical of intact magnetosome chains ${ }^{20}$. Our work demonstrates that the magnetic

323 signatures of these sediments can be explained by the sole presence of MTB producing

324 multi-stranded magnetosome chains.

325 From micromagnetic simulations and FORC measurements, we demonstrated that the

326 main characteristics of unresolved magnetofossil signatures can be reproduced by

327 endmember structures consisting of native single- and double-stranded magnetosome

328 chains, as well as fold-collapsed chains. All simulated structures contribute to the FORC

329 central ridge and produce hysteresis loops with squareness values comprised between

$330 \sim 0.47$ and 0.5. Double-stranded and fold-collapsed chains bear FORC signatures associated

331 with the nucleation and annihilation of FC states where the two strands carry opposite

332 magnetizations. A comparison of double-stranded chain simulations with the topologically 
333 identical necklace structures produced by the AMB-1 mutant strain shows that the elevated

334 hysteresis squareness of magnetofossils is due to the stabilization of SD magnetic states by

335 the strong uniaxial anisotropy of linear chain structures. This anisotropy is responsible for

336 the unique combination of SD and FC characteristics of magnetofossil signatures among

337 known natural sources of magnetite particles. Destruction of the native chain anisotropy,

338 as in magnetosome clusters obtained from laboratory extraction or produced by $\Delta m a m J$

339 mutants of the MSR-1 magnetotactic strain ${ }^{36}$, changes the original magnetic signature in a

340 manner that is incompatible with the hysteresis properties of magnetofossil-rich sediment

341 (Fig. 1), mainly because of an excessive squareness lowering. Complete chain collapse is

342 therefore not expected to occur in sediment. Looped chains, as produced by the AMB-1

343 mutant and by the algorithm used for micromagnetic simulations in ref. ${ }^{4}$, possess

344 intermediate properties between those of native chains and SD particle clusters, which can

345 be explained by the low-dimensionality of such structures. Closed chain loops, such as

346 those produced by the AMB-1 mutant strain, are incompatible with sedimentary signatures

347 (Fig. 1). On the other hand, widely open chain loops with hysteresis squareness values

348 compatible with those of SD particles in sediment [Fig. 5a in ref. ${ }^{38}$ ] resemble bended single-

349 stranded chains used in our simulation. The very subdued FORC contributions above the

350 central ridge generated by these single-stranded chains configurations, however, do not

351 reflect the FORC signature associated with SD-sized magnetite particles in magnetofossil-

352 rich sediments (feature 2 in Fig. 6A). This natural signature, together with hysteresis

353 squareness values close to 0.5 , cannot be obtained from single-stranded chains alone,

354 regardless of how much their native geometry is disrupted by the algorithm used in ref. ${ }^{\text {s, }}$, 
355 and therefore requires significant contributions from native multi-stranded chains or fold-

356 collapsed chains that preserve the native uniaxial anisotropy.

357 Modeling and experiments on the AMB-1 mutant agree, but it is critical to see whether

358 the observed patterns also appear in magnetofossil-rich sediments. A direct comparison

359 between the FORC signatures of simulated chains and of in situ $<1 \mu \mathrm{m}$ particles in a

360 magnetofossil-rich sediment ${ }^{20}$ reveals additional important insights. For this purpose, a

361 composite FORC diagram has been generated using a mixture of the six chain structures

362 simulated in this work (Fig. 6B). The chosen composition is arbitrary, since the natural

363 proportions of equant and prismatic magnetosomes, and possibly also of single- and multi-

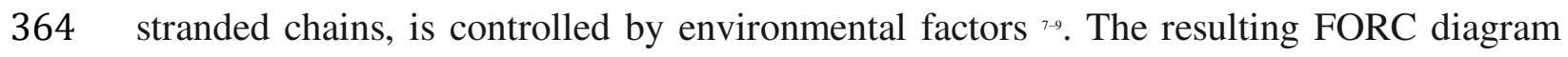

365 shares striking similarities with the natural sediment example, but also important

366 differences. Both diagrams feature a central ridge, but the coercivity range of simulations

367 is comprised between $\sim 20$ and $\sim 130 \mathrm{mT}$, while the natural central ridge contains significant

368 contributions also in the $0-20 \mathrm{mT}$ range. The existence of a minimum magnetofossil

369 coercivity threshold in the simulations is explainable by the uniaxial anisotropy of non-

370 looped chain structures. Immature magnetosomes at the chain ends, which have been

371 included in the simulations, do not change this scenario, since they are strongly coupled to

372 the rest of the chain. Thermal relaxation, which has not been considered in the simulations,

373 tend to reduce the effective coercivity by an amount known as fluctuation field, thus

374 shifting the central ridge toward lower fields ${ }^{4}$. A large coercivity reduction with respect to

375 the simulation results can be excluded because (i) only a very minor fraction of observed

376 magnetofossil sizes fall close to the lower SD stability limit (Fig. S2), as confirmed also

377 by hysteresis squareness values close to 0.5 , and (ii) the vertical offset of the central ridge, 
378 which is comparable with the amplitude of the fluctuation field so, is $\leq 0.5 \mathrm{mT}$ in 379 magnetofossil-bearing sediment ( $\sim .4 \mathrm{mT}$ in Fig. $6 \mathrm{~A})$.

380 The interpretation of the low-coercivity range $(<20 \mathrm{mT})$ of the central ridge is uncertain.

381 Short chain fragments of 1-3 equant magnetosomes possess significantly lower coercivities

382 than fully developed chains ${ }^{38}$, and can therefore contribute to the central ridge over this

383 range. It is not known whether such fragments are produced by MTB under natural, slow-

384 growing conditions, or if longer chains are naturally fragmented through adhesion of

385 segments of the same chain to different sediment particles. Well-dispersed, nearly equidi-

386 mensional SD particles, produced for instance by dissimilatory iron reduction in sediment

$387{ }^{51}$ or during soil formation ${ }^{52}$ can also contribute to the central ridge in the 0-20 $\mathrm{mT}$ range.

388 The existence of low-coercivity contributions in the central ridge of most sediments means

389 that biogenic and inorganic origins of SD magnetite particles, as postulated for the

390 Paleocene-Eocene Thermal Maximum, are not mutually exclusive ${ }^{\text {sss. }}$.

391 Other FORC signatures of our micromagnetic simulations are qualitatively similar to

392 those of magnetofossil-bearing sediments, including the slight asymmetry between the

393 upper and the lower quadrant, and the abrupt $B_{c}$-termination of FC contributions (feature 2

394 in Fig. S6). The contour lines of the simulated FORC are clearly affected by localized

395 contributions of FC nucleation and annihilation of individual chain endmembers, which

396 are not completely merged into a smooth distribution as in the natural counterpart. Small

397 negative amplitudes above the central ridge are also not completely cancelled. This is

398 understandable considering that simulations were limited to few, well-defined geometries.

399 For instance, single- and multi-stranded chains made of bullet- or tooth-shaped magneto-

400 somes were not included. Multi-stranded chains of bullet-shaped magnetosomes are 
401 characterized by similarly elevated hysteresis squareness values as our double-stranded

402 simulations, with a central ridge peaking at $\sim 60 \mathrm{mT}{ }^{s 5}$. This intermediate coercivity range

403 would fill the gaps between simulated chains of equidimensional and prismatic

404 magnetosomes, making the resulting FORC diagram more similar to that of natural

405 magnetofossils.

406 Magnetic fingerprints have become an essential tool for magnetofossil identification (SI

407 appendix). The different FORC signatures of intact chains on the one hand, and fold-

408 collapsed or multi-stranded chains on the other, make the exact magnetic fingerprint of

409 magnetofossils depend, to a certain extent, on the original MTB composition and degree

410 of single-stranded chain preservation, yielding variable proportions of the BS and BH

411 coercivity components ${ }^{34}$, and of central ridge and FC contributions to the FORC diagram

412 (Table 1). This means that PCA analyses of magnetofossil-rich sedimentary sequences

413 deposited under changing environmental conditions likely require more than one

414 endmember to capture the variability of magnetofossil fingerprints. For instance, changes

415 in the proportion of single- and double-stranded chains yield different relative contributions

416 of the central ridge and FC features in the FORC diagrams, resulting in at least two

417 endmembers: one dominated by the signature elements of single-stranded chains (central

418 ridge and negative contributions in the lower quadrant, near the $B_{u}$ axis), and the other

419 dominated by those of multi-stranded chains (FC nucleation and annihilation contributions

420 in the upper and lower quadrant). Variable proportions of single and multi-stranded chains

421 can split the magnetofossil signature into two PCA endmembers that do not necessarily

422 correspond to the physical signature of the two groups: for instance, the central ridge of

423 both types of chains can go into one endmember, and the lobes of two-state chains into 
424 another endmemeber, possibly together with the VO signatures of lithogenic inputs ${ }^{{ }^{6}}$, if

425 these co-vary with the magnetofossil content as in certain pelagic environments ${ }^{8}$. This

426 might explain why some endmembers interpreted as magnetofossils contain the signatures

427 of two-state chains [e.g. EM2 in Figs. 8 and 10 of ref. ${ }^{49}$ ], while other do not [e.g. EM2 in

428 Fig. 11 of ref. ${ }^{57}$.

429 Magnetic measurements have been used to obtain the in situ sedimentary FORC

430 signature of SD magnetite ${ }^{20}$, including non-central ridge contributions that are specific to

431 multi-stranded chains. Such measurements are of paleontological interest and could

432 provide a temporal resolution on the evolution of magnetosome formation in MTB ${ }^{4}$. Only

433 bacteria belonging to the more ancestral Delta-proteobacteria and Nitrospirae phyla were

434 thought to produce multi-stranded chains of magnetosomes ${ }^{58}$. However, they have been

435 now identified in the more recent Alpha-proteobacteria and Gamma-proteobacteria phyla

$436{ }^{59,60 .}$. Therefore, multi-stranded chain configuration has no specific phylogenetic distribution

437 and might instead correspond to an adaptation to specific environments. In MTB,

438 magnetosomes are aligned along cytoskeletal filaments made of actin-like proteins ${ }^{6}$.

439 Among them, the protein MamK is ubiquitously conserved in all known MTB. Bacteria

440 forming multiple magnetosome chains contain several copies of genes encoding actin-like

441 proteins in addition to MamK ${ }^{62}$. We can thus hypothesize that genetic factors are

442 responsible for multi-stranded chain configurations ${ }^{\circ 2}$. They may include gene duplication

443 events ${ }^{39}$, or horizontal gene transfers (i.e. an exchange of genetic material through direct

444 physical interactions between two organisms) ${ }^{63}$. Once the genetic basis of chain

445 configurations is fully understood, the methodology we describe in this work can be used 
446 to provide a temporal constrain on evolutionary events that occurred in one of the oldest

447 and more diverse group of biomineralizing organisms.

\section{Material and methods}

449 Deletion plasmid construction and generation of the $\Delta m a m J \Delta l i m J \Delta M I S A M B-1$

450 mutant strain. Detailed procedures for plasmid construction and AMB-1 transformation

451 are provided in SI appendix.

452 Bacterial cultures. Magnetospirillum magneticum strain AMB-1 (ATCC700264) and

453 mutant AMB-1 strain were cultivated following ATCC recommendations in 500-mL

454 screw-caped bottles until end of the exponential phase. Bottles were filled with $300 \mathrm{~mL}$ of

455 growth medium, and placed in a glove box with controlled atmosphere $\left(10 \% \mathrm{O}_{2}, 90 \% \mathrm{~N}_{2}\right)$

456 at $30^{\circ} \mathrm{C}$ after inoculation (1/100). The sole iron source in bacterial growth medium was

$457 \mathrm{Fe}(\mathrm{III})$-citrate added at $150 \mu \mathrm{M}$.

458 Transmission electron microscopy. Cells were deposited on copper grids coated with

459 a Formvar and carbon films, and characterized with a FEI Tecnai 12 transmission electron

460 microscope operating at $120 \mathrm{kV}$. Magnetite length and width were measured using the

461 ImageJ software.

462 Native magnetic moment measurements. AMB-1 cultures were centrifuged (8 000

$463 \mathrm{rpm}, 10 \mathrm{~min}$ ) and suspended in $10 \mathrm{~mL}$ of phosphate buffer (PBS). $100 \mu \mathrm{L}$ of cell suspension

464 were transferred in plastic vials (diameter of $4 \mathrm{~mm}$ ), and placed in a Lakeshore Micro-Mag

4653900 VSM for acquisition of remanent magnetizations at room temperature. 
466 Off-axis electron holography. Both wild-type and mutant AMB-1 strains were

467 cultivated in 10-mL glass tubes following the protocol described above. Cells were

468 centrifuged (8 $000 \mathrm{rpm}, 10 \mathrm{~min}$ ) and suspended in $100 \mu \mathrm{L}$ of phosphate buffer (PBS). They

469 were then deposited on copper grids coated with an ultra-thin carbon membrane. Off-axis

470 electron holography was carried out using a Hitachi HF3300C microscope operated at 300

$471 \mathrm{kV}$ and equipped with a cold field emission gun and a spherical aberration corrector (CEOS

472 B-Corr). Electron holography experiments were performed in a specific corrected Lorentz

473 mode, allowing a spatial resolution down to $0.5 \mathrm{~nm}$ in a magnetic field-free sample

474 environment ${ }^{64}$. All holograms were recorded in a 2-biprism configuration to avoid artifacts

475 linked to Fresnel fringes and to set separately the interference area size and the fringe

476 spacing ${ }^{6}$. The fringe spacing is equal to $1 \mathrm{~nm}$ (7 pixels) allowing for a spatial resolution

477 of $1.5 \mathrm{~nm}$ for the treated magnetic phase images. The exposure time was set to $1 \mathrm{~nm}$ using

478 dynamic automation acquisition for removing instabilities and applying the fringe $\pi$-shift

479 method ${ }^{6,67}$. Phase and amplitude images were extracted from the holograms by using

480 homemade software based on fast-Fourier transform approach. The magnetic and

481 electrostatic contributions have been separated by acquiring two holograms for which the

482 sample has been switched upside down (flipped $180^{\circ}$ ): the magnetic contribution was

483 obtained by evaluating the difference of the phase images from the two holograms divided

484 by two.

485 Micromagnetic modeling. Micromagnetic modeling has been performed in two steps.

486 First, randomly oriented synthetic chains for each of the six configurations have been

487 generated. Magnetosome-specific control parameters include size, shape, and crystal axes

488 orientation according to realistic distributions obtained from the literature. Chain-specific 
489 control parameters include magnetosome gaps, number of crystals, size tapering, and chain

490 bending within the elastic limit. The configuration of double-stranded chains is additionally

491 controlled by the lag of one strand with respect to the other, and by twisting about a

492 common axis. The distributions of these parameters have been empirically chosen to match

493 examples reported in the literature. Next, micromagnetic calculations have been performed

494 to simulate FORC measurements with a resolution of $1 \mathrm{mT}$. These calculations are based

495 on the minimization of the total magnetic energy resulting from magnetocrystalline and

496 shape anisotropy of individual crystals, the Zeeman energy in the applied field, and the

497 energy of magnetostatic interactions between pairs of crystals. Simulated FORC

498 measurements have been further processed with VARIFORC ${ }^{68}$, using minimal smoothing

499 parameters to eliminate the statistical noise caused by the relatively limited number of 500 simulated chains. Further details are given in the SI Appendix.

502 FORC measurements. AMB-1 cultures were recovered by centrifugation (8 $000 \mathrm{rpm}$,

$50310 \mathrm{~min}$ ). Bacterial pellets were transferred in a $1.5-\mathrm{mL}$ eppendorf tube and dried at room 504 temperature under anoxic conditions in a glove box $\left(\left[\mathrm{O}_{2}\right]<1 \mathrm{ppm}\right)$ to prevent magnetite 505 oxidation. Samples were stored under anoxic conditions until before being measured with 506 a Lakeshore 8600 VSM.

508 ACKNOWLEDGMENTS. AK and MA are supported by grants through the National 509 Science Foundation (1504681) and National Institutes of Health (R35GM127114). MA is 510 supported by grant through the Fondation pour la Recherche Médicale 511 (ARF201909009123). The research leading to these results has received funding from the 
512 European Union Horizon 2020 research and innovation program under grant agreement

513 No. 823717 - ESTEEM3. The authors acknowledge the French National Research Agency

514 under the "Investissement d'Avenir" program reference No. ANR-10-EQPX-38-01 and the

515 "Conseil Régional Midi-Pyrénées" and the European FEDER for financial support within

516 the CPER program. This work was also supported by the international associated

517 laboratory $\mathrm{M}^{2} \mathrm{OZART}$.

518 


\section{References}

520 1. Egli, R., Fabio, F. \& Roberts, A. Introduction to 'Magnetic ironminerals in sediments and their 521 relation to geologic processes, climate, and the geomagnetic field'. Global and Planetary

$522 \quad$ Change 110, 259-263 (2013).

523 2. Fortin, D. \& Langley, S. Formation and occurrence of biogenic iron-rich minerals. Earth-Sci. $524 \quad$ Rev. 72, 1-19(2005).

525 3. Uebe, R. \& Schueler, D. Magnetosome biogenesis in magnetotactic bacteria. Nat. Rev.

$526 \quad$ Microbiol. 14, 621-637 (2016).

527 4. Lin, W. et al. Origin of microbial biomineralization and magnetotaxis during the Archean.

528 Proc. Natl. Acad. Sci. U. S. A. 114, 2171-2176 (2017).

529 5. Simmons, S. L., Sievert, S. M., Frankel, R. B., Bazylinski, D. A. \& Edwards, K. J.

530 Spatiotemporal distribution of marine magnetotactic bacteria in a seasonally stratified coastal 531 salt pond. Appl. Environ. Microbiol. 70, 6230-6239 (2004).

532 6. Flies, C. B. et al. Diversity and vertical distribution of magnetotactic bacteria along chemical 533 gradients in freshwater microcosms. FEMS Microbiol. Ecol. 52, 185-195 (2005).

$5347 . \quad$ Hesse, P. P. Evidence for bacterial palaeoecological origin of mineral magnetic cycles in oxic 535 and sub-oxic Tasman Sea sediments. Mar. Geol. 117, 1-17 (1994).

536 8. Roberts, A. P. et al. Magnetotactic bacterial abundance in pelagic marine environments is 537 limited by organic carbon flux and availability of dissolved iron. Earth Planet. Sci. Lett. 310, $538 \quad 441-452(2011)$.

539 9. Usui, Y., Yamazaki, T. \& Saitoh, M. Changing Abundance of Magnetofossil Morphologies in

540 Pelagic Red Clay Around Minamitorishima, Western North Pacific. Geochemistry,

541 Geophysics, Geosystems 18, 4558-4572 (2017).

542 10. McCausland, H. C. \& Komeili, A. Magnetic genes: Studying the genetics of biomineralization 543 in magnetotactic bacteria. PLOS Genetics 16, e1008499 (2020).

544 11. Petersen, N., von Dobeneck, T. \& Vali, H. Fossil bacterial magnetite in deep-sea sediments

545 from the South Atlantic Ocean. Nature 320, 611-615 (1986). 
546 12. Kopp, R. E. \& Kirschvink, J. L. The identification and biogeochemical interpretation of fossil

547 magnetotactic bacteria. Earth-Sci. Rev. 86, 42-61 (2008).

548 13. Larrasoana, J. C. et al. Paleomagnetic and paleoenvironmental implications of magnetofossil 549 occurrences in late Miocene marine sediments from the Guadalquivir Basin, SW Spain. Front. $550 \quad$ Microbiol. 5, 71 (2014).

551 14. Ouyang, T. et al. Variable remanence acquisition efficiency in sediments containing biogenic 552 and detrital magnetites: Implications for relative paleointensity signal recording. Geochem. 553 Geophys. Geosyst. 15, 2780-2796 (2014).

554 15. Roberts, A. P. et al. Resolving the Origin of Pseudo-Single Domain Magnetic Behavior.

555 Journal of Geophysical Research: Solid Earth 122, 9534-9558 (2017).

556 16. Lascu, I., Einsle, J. F., Ball, M. R. \& Harrison, R. J. The Vortex State in Geologic Materials:

557 A Micromagnetic Perspective. Journal of Geophysical Research: Solid Earth 123, 7285-7304

$558 \quad$ (2018).

559 17. Egli, R., Chen, A. P., Winklhofer, M., Kodama, K. P. \& Horng, C.-S. Detection of

560 noninteracting single domain particles using first-order reversal curve diagrams. Geochem.

561 Geophys. Geosyst. 11, Q01Z11 (2010).

562 18. Roberts, A. P., Chang, L., Heslop, D., Florindo, F. \& Larrasoaña, J. C. Searching for single

563 domain magnetite in the "pseudo-single-domain" sedimentary haystack: Implications of

564 biogenic magnetite preservation for sediment magnetism and relative paleointensity

565 determinations. J. Geophys. Res.-Solid Earth 117, (2012).

566 19. Heslop, D., Roberts, A. P. \& Chang, L. Characterizing magnetofossils from first-order reversal 567 curve (FORC) central ridge signatures. Geochemistry, Geophysics, Geosystems 15, 2170-2179 $568 \quad$ (2014).

569 20. Ludwig, P. et al. Characterization of primary and secondary magnetite in marine sediment by 570 combining chemical and magnetic unmixing techniques. Global and Planetary Change 110, $571 \quad 321-339(2013)$. 
572

573

574

575

576

577

578

579

580

581

582

583

584

585

586

587

588

589

590

591

592

593

594

595

596

597

21. Muxworthy, A., Williams, W. \& Virdee, D. Effect of magnetostatic interactions on the hysteresis parameters of single-domain and pseudo-single-domain grains. J. Geophys. Res.Solid Earth 108, (2003).

22. Dumas, R. K., Li, C.-P., Roshchin, I. V., Schuller, I. K. \& Liu, K. Magnetic fingerprints of sub-100 nm Fe dots. Phys. Rev. B 75, 134405 (2007).

23. Kobayashi, A. et al. Experimental observation of magnetosome chain collapse in magnetotactic bacteria: Sedimentological, paleomagnetic, and evolutionary implications. Earth Planet. Sci. Lett. 245, 538-550 (2006).

24. Li, J., Wu, W., Liu, Q. \& Pan, Y. Magnetic anisotropy, magnetostatic interactions and identification of magnetofossils. Geochem. Geophys. Geosyst. 13, Q10Z51 (2012).

25. Amor, M., Mathon, F. P., Monteil, C. L., Busigny, V. \& Lefevre, C. T. Iron-biomineralizing organelle in magnetotactic bacteria: function, synthesis and preservation in ancient rock samples. Environ. Microbiol. 22, 3611-3632 (2020).

26. Shcherbakov, V. P., Winklhofer, M., Hanzlik, M. \& Petersen, N. Elastic stability of chains of magnetosomes in magnetotactic bacteria. Eur. Biophys. J. Biophy. 4, 319-326 (1997).

27. Hanzlik, M., Winklhofer, M. \& Petersen, N. Pulsed-field-remanence measurements on individual magnetotactic bacteria. J. Magn. Magn. Mater. 248, 258-267 (2002).

28. Varón, M. et al. Dipolar Magnetism in Ordered and Disordered Low-Dimensional Nanoparticle Assemblies. Sci Rep 3, 1234 (2013).

29. Carvallo, C., Dunlop, D. J. \& Özdemir, Ö. Experimental comparison of FORC and remanent Preisach diagrams. Geophys. J. Int. 162, 747-754 (2005).

30. Pan, Y. X. et al. Rock magnetic properties of uncultured magnetotactic bacteria. Earth Planet. Sci. Lett. 237, 311-325 (2005).

31. Muxworthy, A. R. \& Williams, W. Critical single-domain/multidomain grain sizes in noninteracting and interacting elongated magnetite particles: Implications for magnetosomes. J. Geophys. Res.-Solid Earth 111, (2006). 
32. Newell, A. J. Transition to superparamagnetism in chains of magnetosome crystals.

599 Geochemistry, Geophysics, Geosystems 10, (2009).

600 33. Newell, A. J. A high-precision model of first-order reversal curve (FORC) functions for single-

601 domain ferromagnets with uniaxial anisotropy. Geochemistry, Geophysics, Geosystems 6, 602 (2005).

603 34. Egli, R. Characterization of Individual Rock Magnetic Components by Analysis of Remanence

604 Curves, 1. Unmixing Natural Sediments. Studia Geophys. Geod. 48, 391-446 (2004).

605 35. Chen, A. P. et al. Magnetic properties of uncultivated magnetotactic bacteria and their

606 contribution to a stratified estuary iron cycle. Nat. Commun. 5, 4797 (2014).

607 36. Katzmann, E. et al. Analysis of Magnetosome Chains in Magnetotactic Bacteria by Magnetic

608 Measurements and Automated Image Analysis of Electron Micrographs. Appl. Environ. 609 Microbiol. 79, 7755-7762 (2013).

610 37. Li, J. et al. Crystal growth of bullet-shaped magnetite in magnetotactic bacteria of the

$611 \quad$ Nitrospirae phylum. J. R. Soc. Interface 12, 20141288 (2015).

612 38. Chang, L., Harrison, R. J. \& Berndt, T. A. Micromagnetic simulation of magnetofossils with 613 realistic size and shape distributions: Linking magnetic proxies with nanoscale observations 614 and implications for magnetofossil identification. Earth Planet. Sci. Lett. 527, 115790 (2019).

615 39. Murat, D., Quinlan, A., Vali, H. \& Komeili, A. Comprehensive genetic dissection of the 616 magnetosome gene island reveals the step-wise assembly of a prokaryotic organelle. Proc. $617 \quad$ Natl. Acad. Sci. U. S. A. 107, 5593-5598 (2010).

618 40. Rioux, J.-B. et al. A Second Actin-Like MamK Protein in Magnetospirillum magneticum

619 AMB-1 Encoded Outside the Genomic Magnetosome Island. PLOS ONE 5, e9151 (2010).

620 41. Abreu, N. et al. Interplay between Two Bacterial Actin Homologs, MamK and MamK-Like,

621 Is Required for the Alignment of Magnetosome Organelles in Magnetospirillum magneticum

622 AMB-1. J. Bacteriol. 196, 3111-3121 (2014).

623 42. Scheffel, A. et al. An acidic protein aligns magnetosomes along a filamentous structure in 624 magnetotactic bacteria. Nature 440, 110-114 (2006). 
625 43. Draper, O. et al. MamK, a bacterial actin, forms dynamic filaments in vivo that are regulated

626 by the acidic proteins MamJ and LimJ. Mol. Microbiol. 82, 342-354 (2011).

627 44. Le Nagard, L. et al. Magnetite magnetosome biomineralization in Magnetospirillum

628 magneticum strain AMB-1: A time course study. Chem. Geol. 530, 119348 (2019).

629 45. Dunin-Borkowski, R. E. et al. Magnetic microstructure of magnetotactic bacteria by electron

630 holography. Science 282, 1868-1870 (1998).

631 46. Lanci, L. \& Kent, D. V. Forward Modeling of Thermally Activated Single-Domain Magnetic 632 Particles Applied to First-Order Reversal Curves. Journal of Geophysical Research: Solid $633 \quad$ Earth 123, 3287-3300 (2018).

634 47. Philipse, A. P. \& Maas, D. Magnetic colloids from magnetotactic bacteria: Chain formation 635 and colloidal stability. Langmuir 18, 9977-9984 (2002).

636 48. Harrison, R. J. \& Lascu, I. FORCulator: A micromagnetic tool for simulating first-order

637 reversal curve diagrams. Geochemistry, Geophysics, Geosystems 15, 4671-4691 (2014).

638 49. Lascu, I. et al. Magnetic unmixing of first-order reversal curve diagrams using principal 639 component analysis. Geochemistry, Geophysics, Geosystems 16, 2900-2915 (2015).

640 50. Berndt, T. A., Chang, L., Wang, S. \& Badejo, S. Time-Asymmetric FORC Diagrams: A New 641 Protocol for Visualizing Thermal Fluctuations and Distinguishing Magnetic Mineral Mixtures. 642 Geochemistry, Geophysics, Geosystems 19, 3056-3070 (2018).

643 51. Moskowitz, B., Frankel, R. \& Bazylinski, D. Rock Magnetic Criteria for the Detection of 644 Biogenic Magnetite. Earth Planet. Sci. Lett. 120, 283-300 (1993).

645 52. Geiss, C. E., Egli, R. \& Zanner, C. W. Direct estimates of pedogenic magnetite as a tool to 646 reconstruct past climates from buried soils. J. Geophys. Res.-Solid Earth 113, (2008).

647 53. Kent, D. V. et al. A case for a comet impact trigger for the Paleocene/Eocene thermal maximum 648 and carbon isotope excursion. Earth Planet. Sci. Lett. 211, 13-26 (2003).

649 54. Lippert, P. C. \& Zachos, J. C. A biogenic origin for anomalous fine-grained magnetic material 650 at the Paleocene-Eocene boundary at Wilson Lake, New Jersey. Paleoceanography 22, 651 PA4104 (2007). 
652 55. Li, J. et al. Biomineralization, crystallography and magnetic properties of bullet-shaped 653 magnetite magnetosomes in giant rod magnetotactic bacteria. Earth and Planetary Science $654 \quad$ Letters 293, 368-376 (2010).

655 56. Chang, L. et al. Widespread occurrence of silicate-hosted magnetic mineral inclusions in 656 marine sediments and their contribution to paleomagnetic recording. Journal of Geophysical 657 Research: Solid Earth 121, 8415-8431 (2016).

658 57. Channell, J. E. T. et al. Magnetic record of deglaciation using FORC-PCA, sortable-silt grain 659 size, and magnetic excursion at $26 \mathrm{ka}$, from the Rockall Trough (NE Atlantic). Geochemistry, 660 Geophysics, Geosystems 17, 1823-1841 (2016).

661 58. Deng, A. et al. In vitro assembly of the bacterial actin protein MamK from ' Candidatus 662 Magnetobacterium casensis' in the phylum Nitrospirae. Protein Cell 7, 267-280 (2016).

663 59. Taoka, A., Kondo, J., Oestreicher, Z. \& Fukumori, Y. Characterization of uncultured giant rod664 shaped magnetotactic Gammaproteobacteria from a freshwater pond in Kanazawa, Japan. 665 Microbiology, 160, 2226-2234 (2014).

666 60. Zhang, H. et al. Magnetotactic Coccus Strain SHHC-1 Affiliated to Alphaproteobacteria Forms 667 Octahedral Magnetite Magnetosomes. Front. Microbiol. 8, (2017).

668 61. Müller, F. D., Schüler, D. \& Pfeiffer, D. A Compass To Boost Navigation: Cell Biology of 669 Bacterial Magnetotaxis. Journal of Bacteriology 202, (2020).

670 62. Kolinko, S., Richter, M., Gloeckner, F.-O., Brachmann, A. \& Schueler, D. Single-cell 671 genomics of uncultivated deep-branching magnetotactic bacteria reveals a conserved set of 672 magnetosome genes. Environ. Microbiol. 18, 21-37 (2016).

673 63. Monteil, C. L. et al. Repeated horizontal gene transfers triggered parallel evolution of 674 magnetotaxis in two evolutionary divergent lineages of magnetotactic bacteria. ISME J. 14, $675 \quad 1783-1794(2020)$.

676 64. Snoeck, E. et al. Off-Axial Aberration Correction using a B-COR for Lorentz and HREM 677 Modes. Microscp. Microanal. 20, 932-933 (2014). 
678 65. Harada, K., Tonomura, A., Togawa, Y., Akashi, T. \& Matsuda, T. Double-biprism electron 679 interferometry. Appl. Phys. Lett. 84, 3229-3231 (2004).

680 66. Gatel, C., Dupuy, J., Houdellier, F. \& Hÿtch, M. J. Unlimited acquisition time in electron 681 holography by automated feedback control of transmission electron microscope. Appl. Phys. 682 Lett. 113, 133102 (2018).

683 67. Volkov, V. V., Han, M. G. \& Zhu, Y. Double-resolution electron holography with simple 684 Fourier transform of fringe-shifted holograms. Ultramicroscopy 134, 175-184 (2013).

685 68. Egli, R. VARIFORC: An optimized protocol for calculating non-regular first-order reversal 686 curve (FORC) diagrams. Global and Planetary Change 110, 302-320 (2013).

687 


\section{Tables}

689 Table 1. Summary of measured and modelled magnetic properties of MTB and magneto690 fossils. $\chi_{\text {ARM }} / \mathrm{IRM}$ is the so-called ARM ratio, and $M_{\mathrm{c}} / M_{\mathrm{s}}$ is the ratio between the magneti691 zation associated by the central ridge and the saturation remanent magnetization (see SI 692 Appendix S2).

\begin{tabular}{|c|c|c|c|c|}
\hline Structure & Reference & $M_{r} / M_{s}$ & $\begin{array}{l}\chi_{\text {ARu }} / \text { IRM } \\
(\mathrm{mm} / \mathrm{A})\end{array}$ & $M_{\mathrm{c}} / \boldsymbol{M}_{\mathrm{r}}$ \\
\hline Sediment $(<1 \mu \mathrm{m})$ & ${ }^{20}$ & 0.47 & $2-3$ & 0.65 \\
\hline \multicolumn{5}{|l|}{ Single-stranded } \\
\hline Cultured MTB & 24,51 & 0.50 & $3.1-3.7$ & 0.65 \\
\hline AMB-1 & This study, ${ }^{24}$ & $0.48-0.50$ & 1.38 & 0.79 \\
\hline Simulated & This study & $0.49-0.50$ & - & $0.71-0.72$ \\
\hline Simulated (random walk) & ${ }_{38}$ & $0.32-0.38$ & - & $?$ \\
\hline \multicolumn{5}{|l|}{ Multi-stranded } \\
\hline MTB (multi-stranded) & ${ }^{30}$ & $0.47-0.51$ & $0.5-0.8$ & $?$ \\
\hline Simulated (2 strands native) & This study & $0.47-0.49$ & - & $0.40-0.42$ \\
\hline Simulated (fold-collapsed) & This study & $0.47-0.50$ & - & $0.37-0.38$ \\
\hline \multicolumn{5}{|l|}{ Clumps and loops } \\
\hline Extracted magnetosomes & ${ }^{24}$ & 0.22 & 0.23 & $?$ \\
\hline M. gyphiswaldense $\Delta m a m J$ & 36 & 0.23 & - & $?$ \\
\hline AMB-1 mutant & This study & 0.31 & - & 0.53 \\
\hline
\end{tabular}

693

694 


\section{Figure captions}

696 Fig. 1. $B_{\mathrm{c}} / B_{\mathrm{c}}$ vs. $M_{\mathrm{s}} / M_{\mathrm{s}}$ diagram showing the hysteresis properties of intact cultured cells 697 2430.51,, SD particles in a magnetofossil-rich sediment ${ }^{20}$, the AMB-1 mutant producing looped 698 magnetosome arrangements (this work), and a $\Delta m a m J$ mutant producing magnetosome 699 clusters ${ }^{36}$. The properties of extracted magnetosomes ${ }^{24}$, micromagnetically simulated 700 chains with increasing degree of looping ${ }^{38}$, and our micromagnetic simulations of intact 701 and fold-collapsed chains are shown for comparison. Departures from $M_{\mathrm{s}} / M_{\mathrm{s}}=0.5$ for ideal, 702 non-interacting, uniaxial SD particles (cultured cells), form trends with increasing slopes, 703 from intact and fold-collapsed configurations with strong uniaxial anisotropy (intact \& 704 folded) to complete randomization (extracted magnetosomes), through the intermediate 705 trend of looped chains (looped).

\section{6}

707 Fig. 2. Micromagnetic simulations of single-stranded, double-stranded, and fold-collapsed 708 chains of equant and prismatic magnetosomes, respectively. For each configuration, the 709 FORC diagram (top) and the corresponding coercivity distributions (bottom, thick lines)

710 are shown. FORC diagrams share the same color scale with contours at $\pm 2, \pm 5,10,20,60$,

71160 , and $80 \%$ of the maximum amplitude. The inset in each FORC diagram shows a 712 representative simulated chain example. Coercivity distributions represented by thick lines 713 correspond, from smallest to largest amplitude, to the central ridge $\left(f_{\text {cr }}\right.$, red $)$, the irreversible 714 hysteresis ( $f_{\text {irr, }}$ green), and the DC demagnetization curve ( $f_{\text {dcd }}$, dashed), respectively. 715 Coercivity distributions represented by thin grey lines correspond to examples of BS (A$716 \mathrm{C})$ and $\mathrm{BS}(\mathrm{D}-\mathrm{F})$ coercivity components obtained from AF demagnetization curves of 717 ARM, after ref. ${ }^{34}$. 
718 Fig. 3. Electron microscopy images of wild-type and mutant AMB-1 cells.

719

720 Fig. 4. Native magnetic states of wild-type AMB-1 and the AMB-1 mutant. Magnetization 721 of a suspension of (A) wild-type and (D) mutant AMB-1 cells in small fields varying

722 between $\pm 2 \mathrm{mT}$, before (black) and after (gray) saturation in a $200 \mathrm{mT}$ field. Electron 723 microscopy and corresponding magnetic phase contours determined by off-axis electron 724 holography images of (B-C) wild-type and (E-F) mutant magnetite chains.

725 
726 Fig. 5. Magnetic properties of wild-type AMB-1 and the AMB-1 mutant. (A-B) FORC 727 diagrams normalized by the saturation magnetization, with same logarithmically spaced 728 contours. Insets are schematic representations of the magnetization of an individual cell as 729 a function of the applied field. Numbers highlight the following features: 1 - central ridge 730 created by the magnetization jump through which the lowest curve merges with curve I; 2 731 - positive and negative contributions from reversible magnetic moment rotation (difference 732 between the slopes of consecutive curves); 3 - signatures of magnetically viscous 733 particles (vertical ridge and central ridge extending to the origin); 4 - nucleation of a 734 negative FC state from a negative SD state (contribution of the first magnetization jump in 735 curve III to the difference between curves II and III); 5 - annihilation of a positive FC 736 state (contribution of the magnetization jump in curve II to the difference between curves 737 I and II); 6 - same as 5, but for the difference between curve II and III; 7 - annihilation 738 of a negative FC state (contribution of the second magnetization jump in curve III to the 739 difference between curves II and III). (C-D) Coercivity distributions of wild-type AMB-1 740 and the AMB-1 mutant, obtained from subsets of FORC data: $f_{\text {ir }}$ - irreversible component 741 of the ascending hysteresis branch, $f_{\mathrm{\alpha d}}$ - DC demagnetization of $M_{\mathrm{s}}, f_{\mathrm{cr}}$ - central ridge.

742 The shaded area represents the $f_{\text {ir }}$ contribution of SD magnetic states that cannot exist in a 743 null field. 
744 Fig. 6. Comparison between the FORC signature of SD particles in a magnetofossil-rich 745 sediment and synthetic results. (a) FORC diagram of in-situ $<1 \mu \mathrm{m}$ magnetite particles 746 (mostly SD) in a pelagic sediment [data from ref. ${ }^{20}$ ]. (b) FORC diagram of a synthetic 747 composite obtained from the six micromagnetically modelled chain structures, with the 748 following relative contributions to the saturation magnetization, chosen for a visual match 749 with (a): $21 \%$ (single-stranded chains of equant magnetosomes), 4\% (single-stranded 750 chains of prismatic magnetosomes), $42 \%$ (native double-stranded chains of equant 751 magnetosomes), $8 \%$ (native double-stranded chains of prismatic magnetosomes), $21 \%$ 752 (fold-collapsed chains of equant magnetosomes), 4\% (fold-collapsed chains of prismatic 753 magnetosomes). The same field range and contour levels with respect to the maximum 754 amplitude are used in both plots to ease the comparison. Numbers highlight the following 755 features: 1 - nucleation/annihilation of FC states, 2 - high-field limit of FC state 756 nucleation/annihilation (asymmetric about the central ridge), 3 - residual negative 757 amplitude from the annihilation of positive FC states, uncompensated by positive ampli758 tudes from intermediate coercivity contributions.

759 


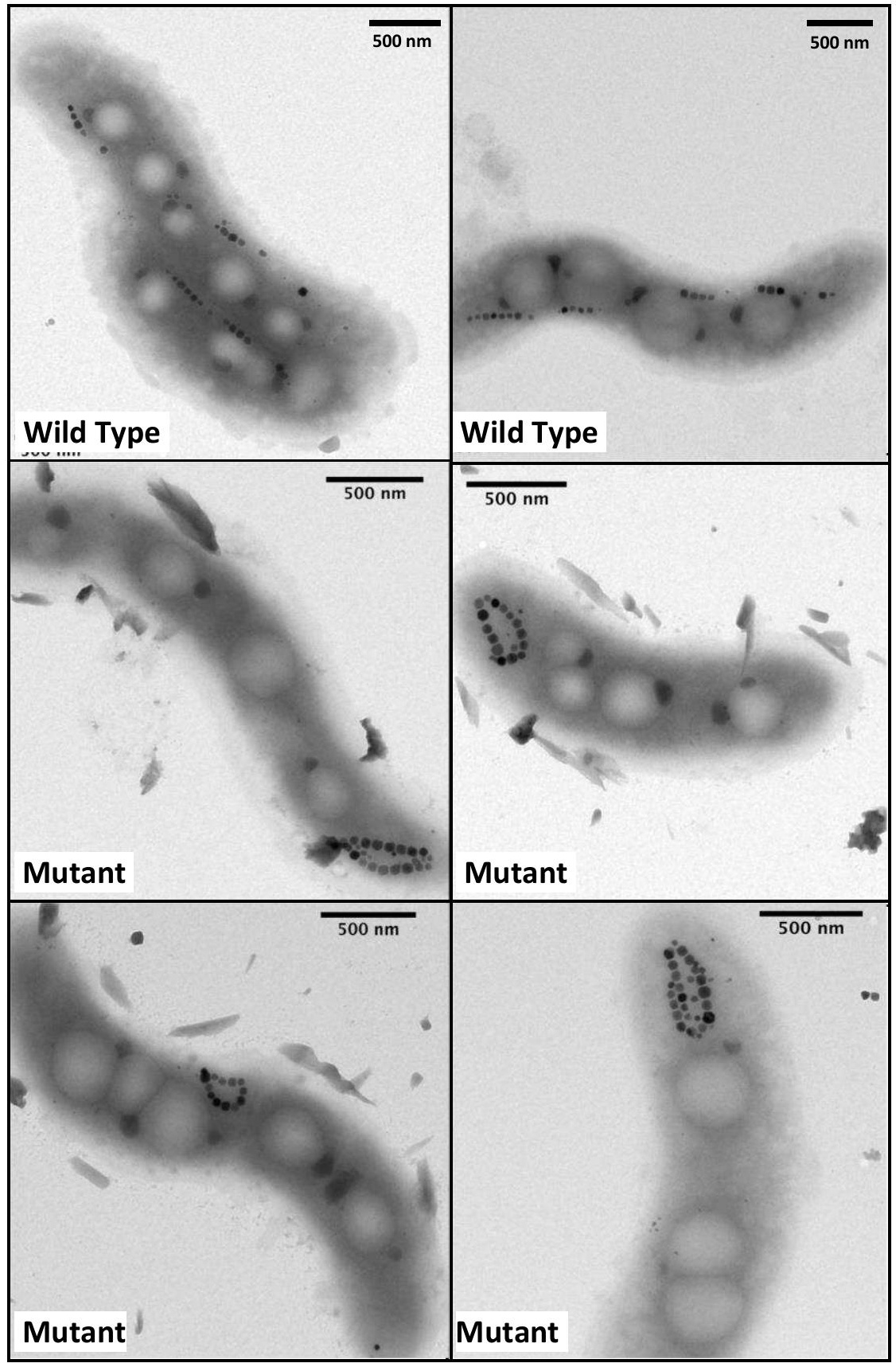




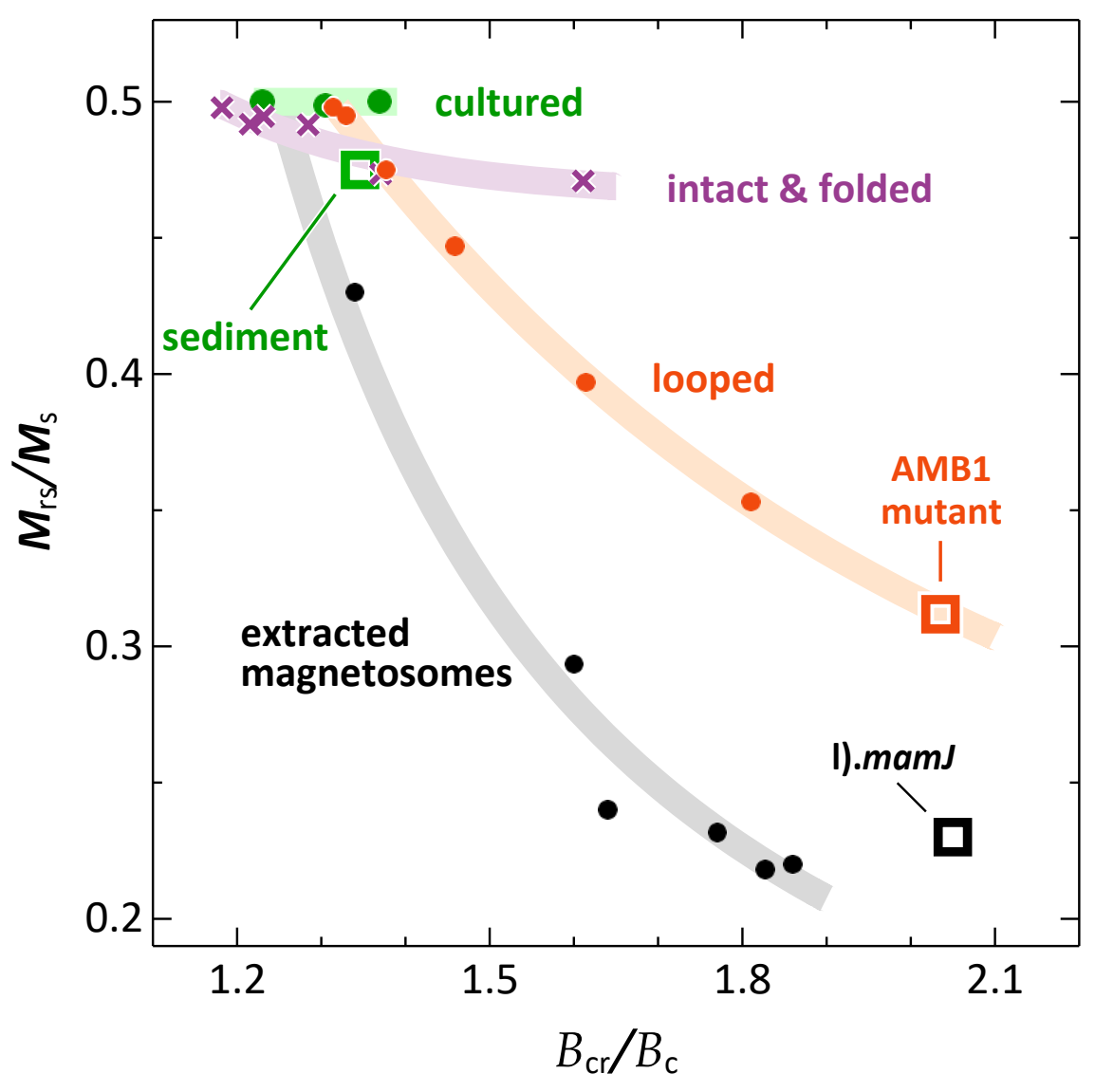



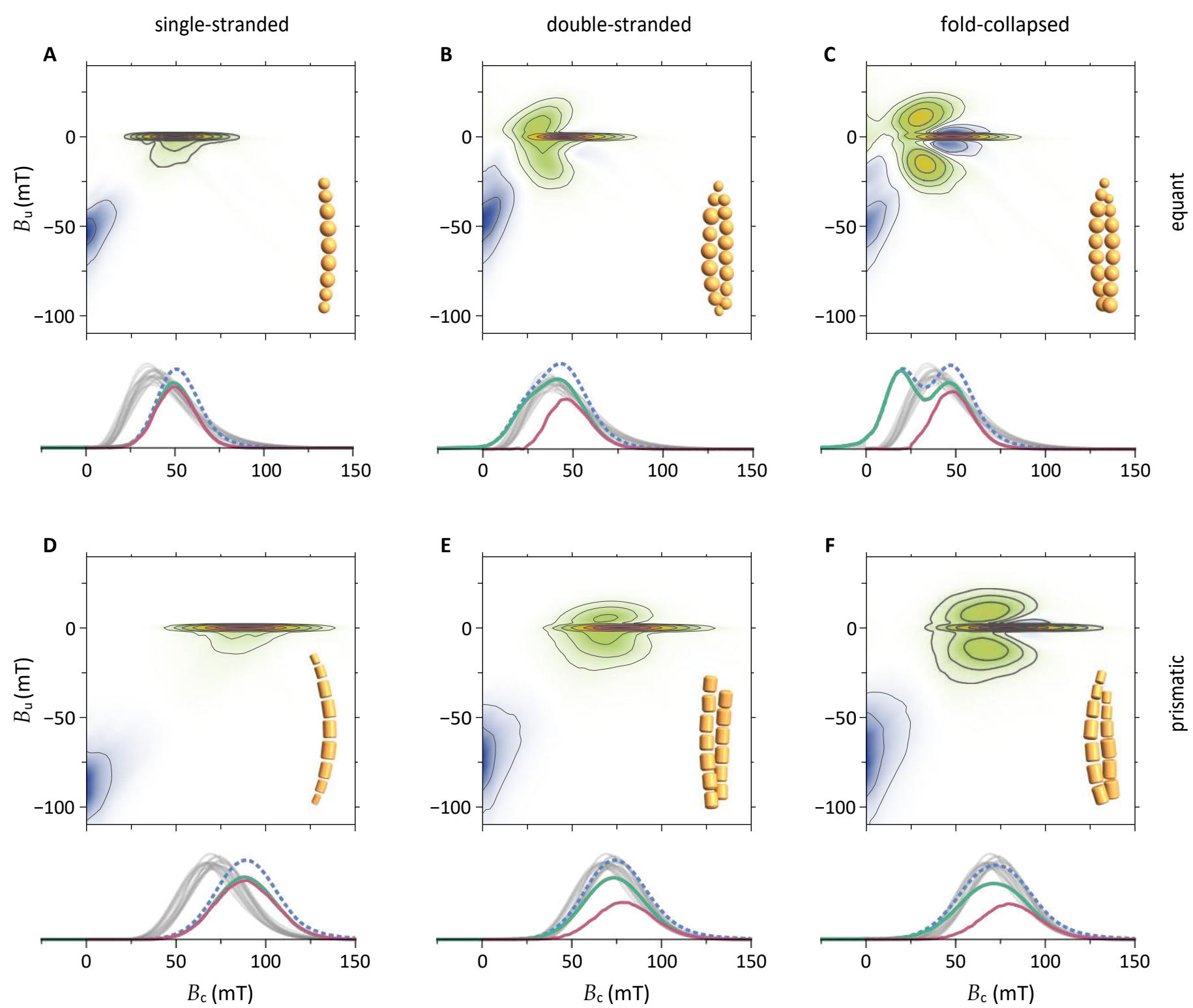

$\frac{.0}{\frac{0}{0}}$ 

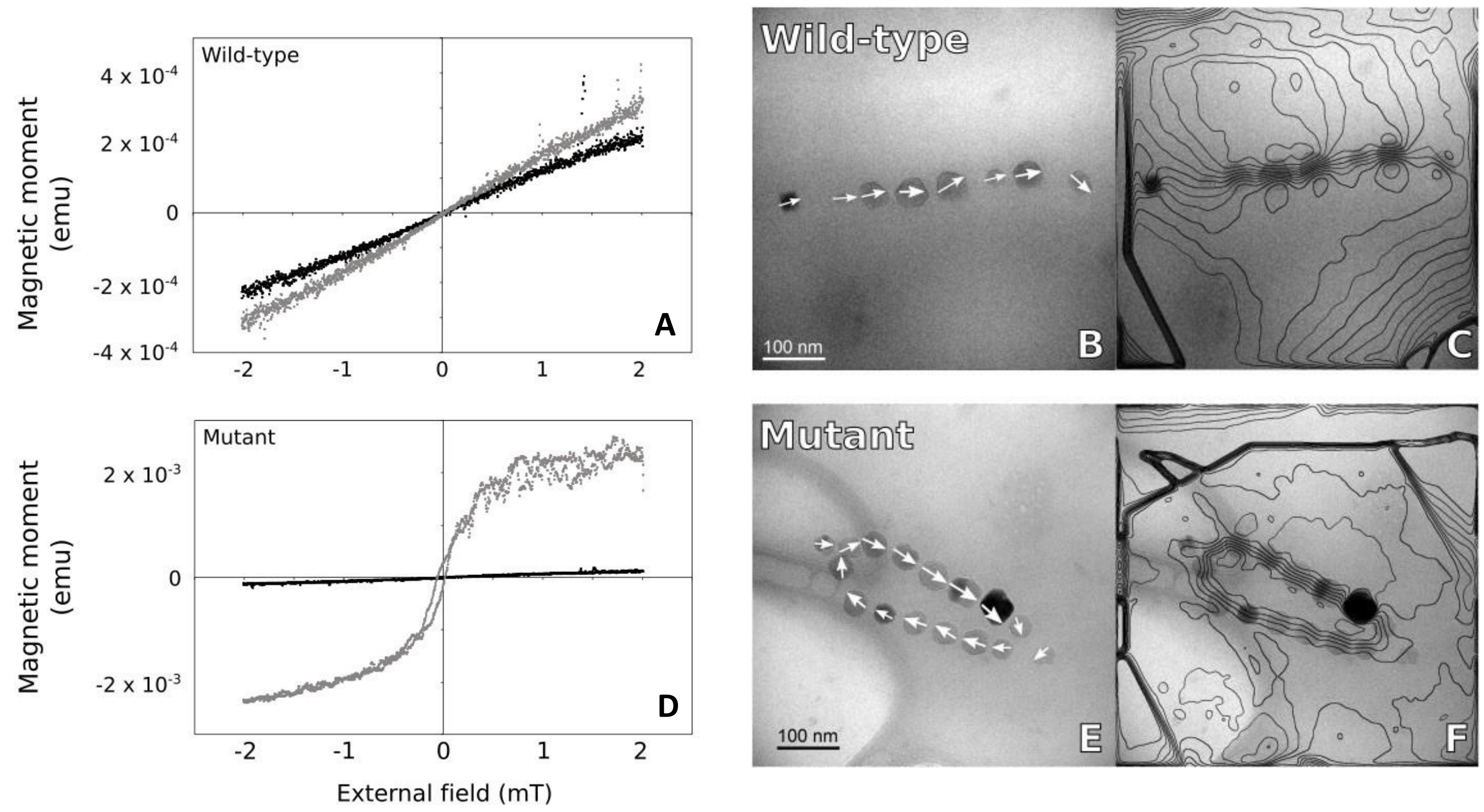

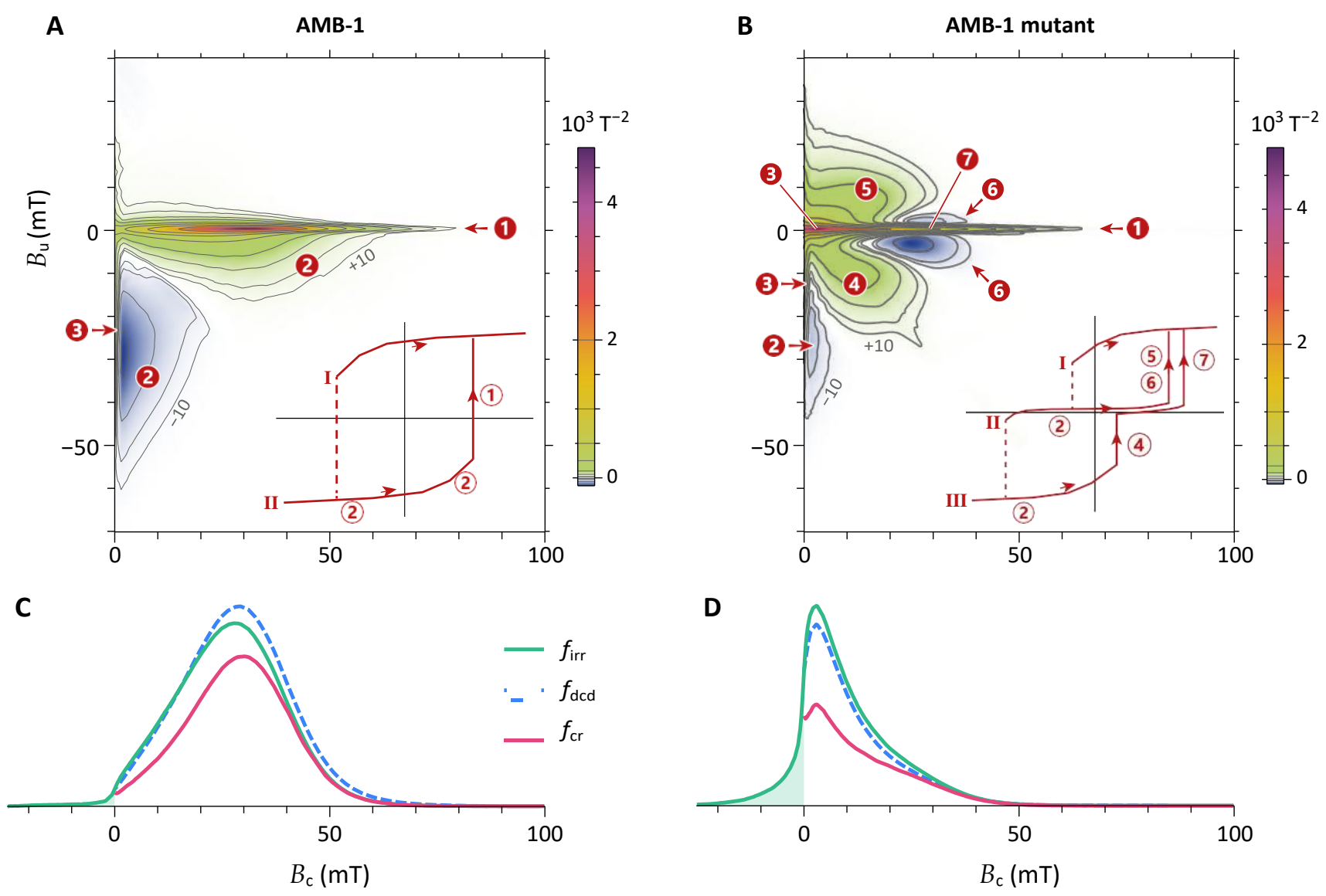

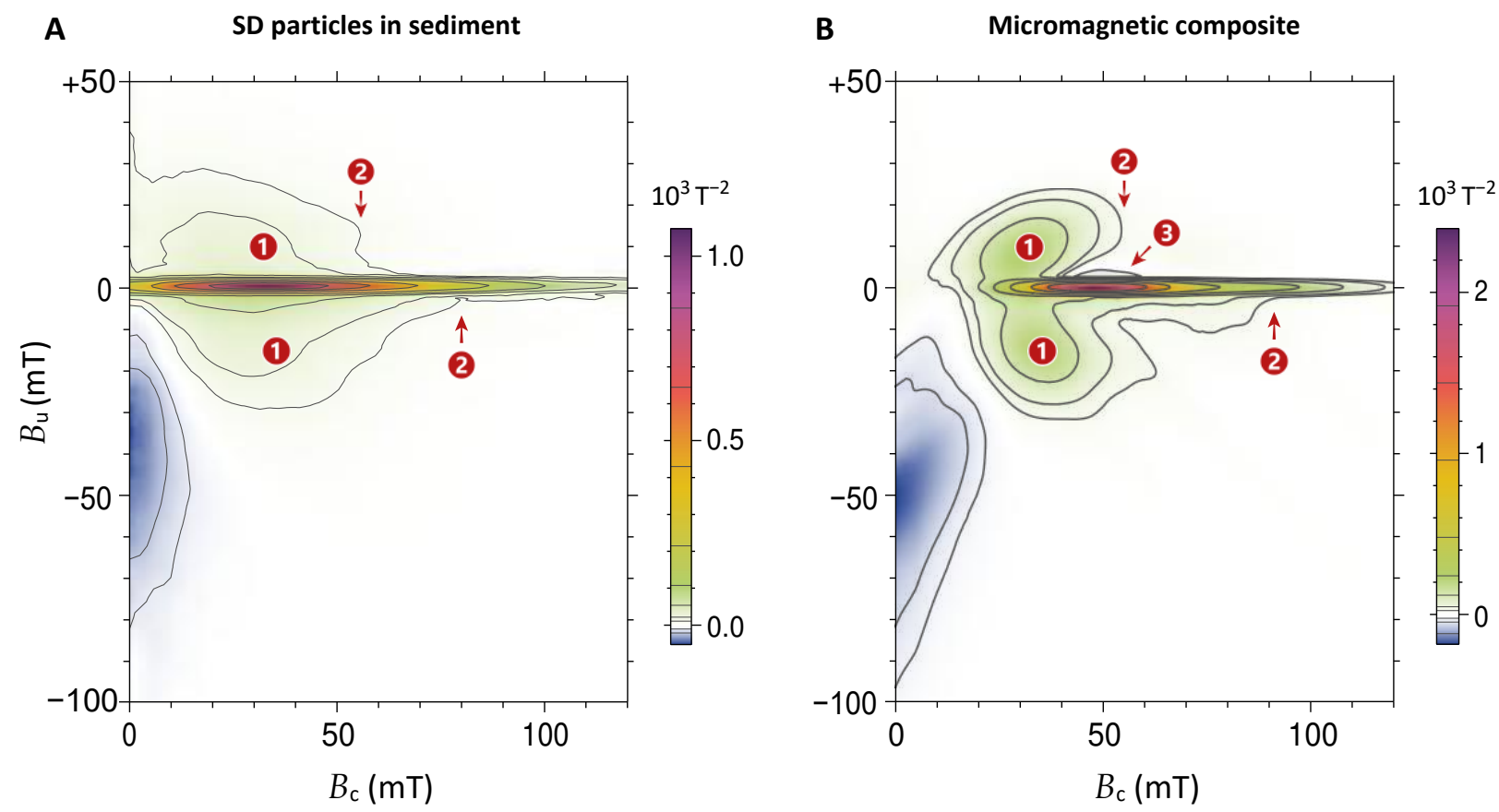
Figures

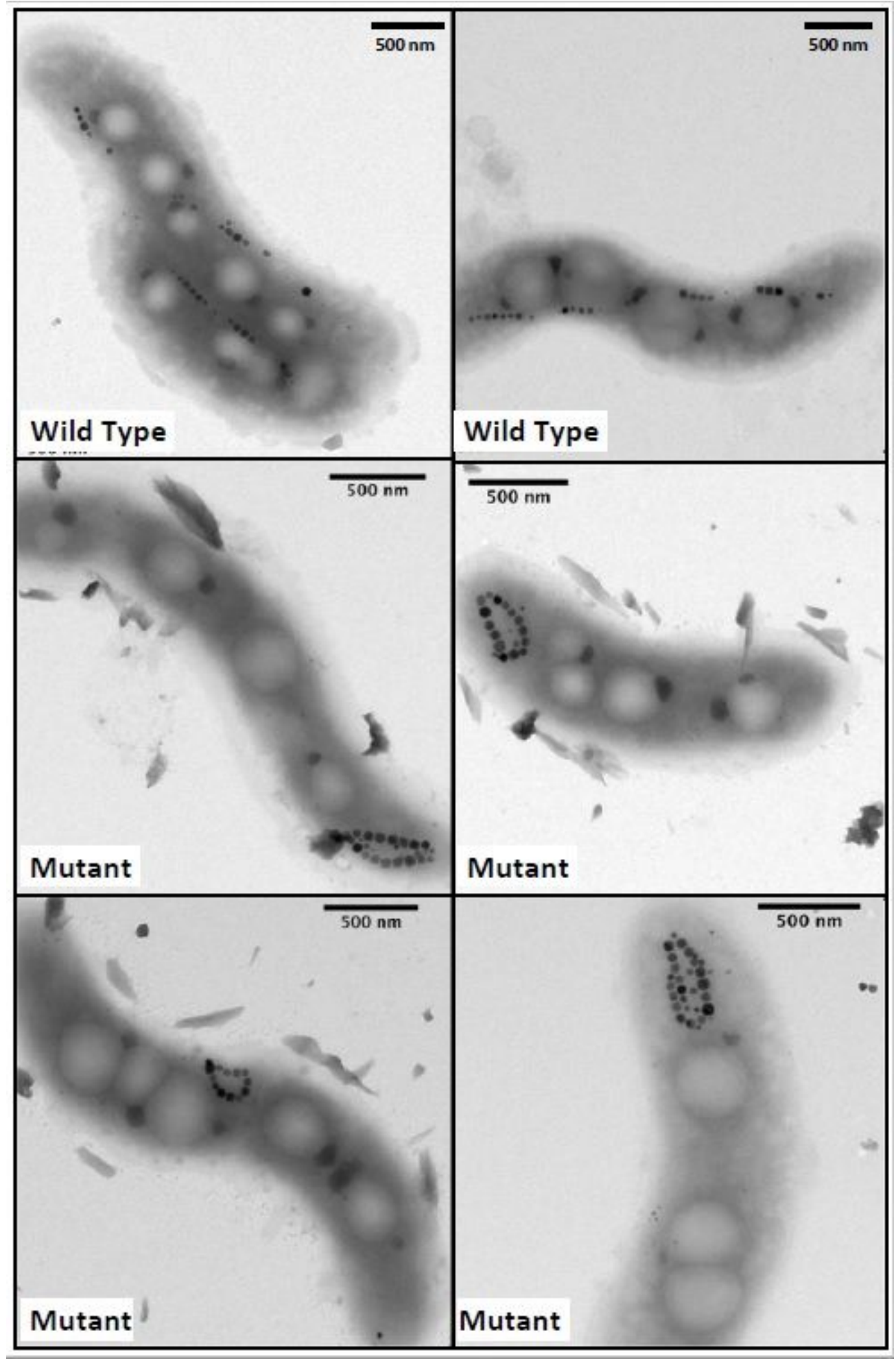

\section{Figure 1}

$\mathrm{Bcr} / \mathrm{Bc}$ vs. Mrs/Ms diagram showing the hysteresis properties of intact cultured cells $24,30,51, \mathrm{SD}$ particles in a magnetofossil-rich sediment 20, the AMB-1 mutant producing looped magnetosome arrangements (this work), and a $\Delta$ mamJ mutant producing magnetosome clusters 36 . The properties of 
extracted magnetosomes 24, micromagnetically simulated chains with increasing degree of looping 38 , and our micromagnetic simulations of intact and fold-collapsed chains are shown for comparison.

Departures from Mrs/Ms $=0.5$ for ideal, non-interacting, uniaxial SD particles (cultured cells), form trends with increasing slopes, from intact and fold-collapsed configurations with strong uniaxial anisotropy (intact \& folded) to complete randomization (extracted magnetosomes), through the intermediate trend of looped chains (looped).

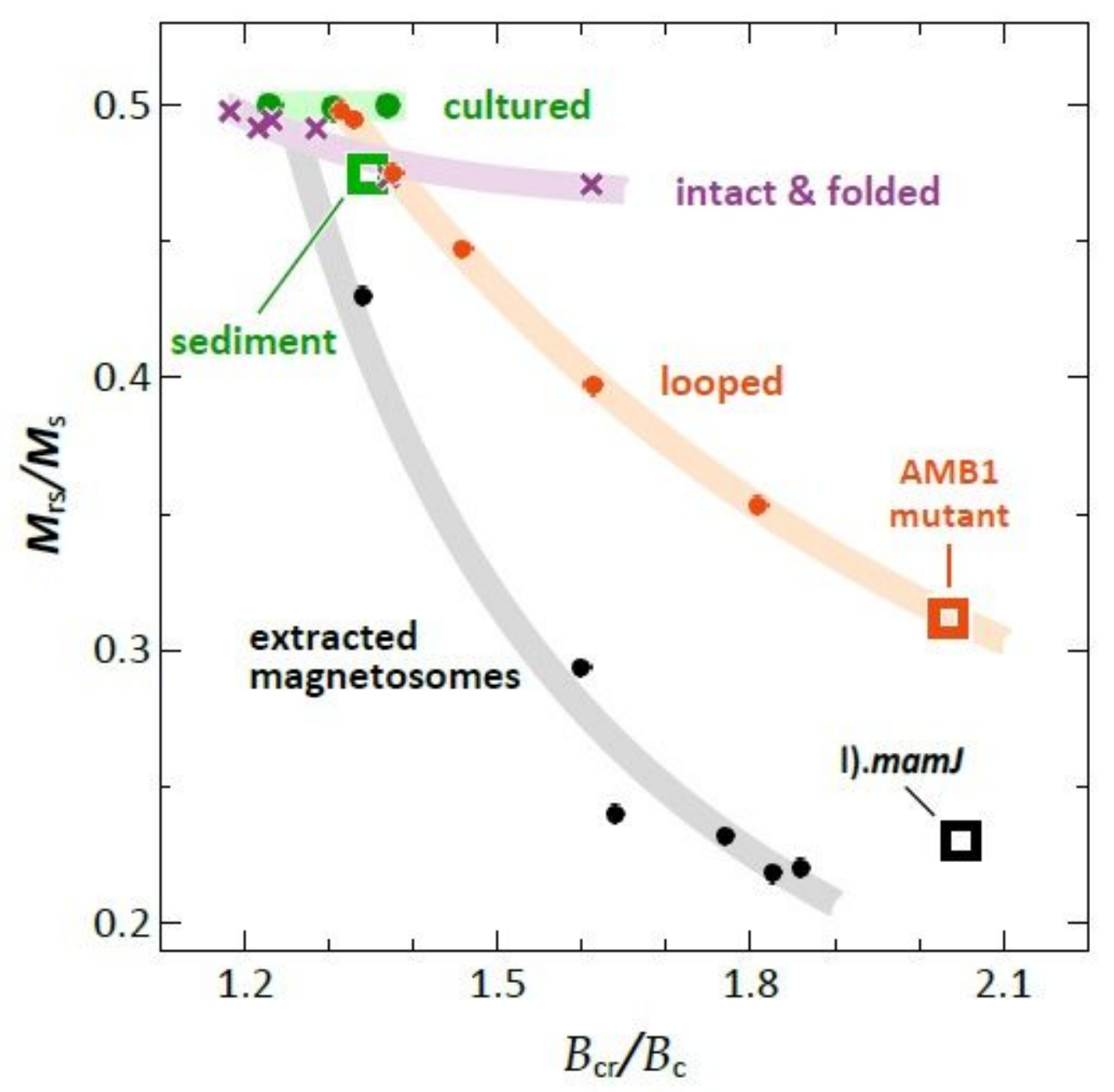

Figure 2

Micromagnetic simulations of single-stranded, double-stranded, and fold-collapsed chains of equant and prismatic magnetosomes, respectively. For each configuration, the FORC diagram (top) and the corresponding coercivity distributions (bottom, thick lines) are shown. FORC diagrams share the same color scale with contours at $\pm 2, \pm 5,10,20,60,60$, and $80 \%$ of the maximum amplitude. The inset in each FORC diagram shows a representative simulated chain example. Coercivity distributions represented by thick lines correspond, from smallest to largest amplitude, to the central ridge ( $\mathrm{fcr}$, red), the irreversible hysteresis (firr, green), and the DC demagnetization curve (fdcd, dashed), respectively. Coercivity distributions represented by thin grey lines correspond to examples of BS (A-C) and BS (D-F) coercivity components obtained from AF demagnetization curves of ARM, after ref. 34 . 

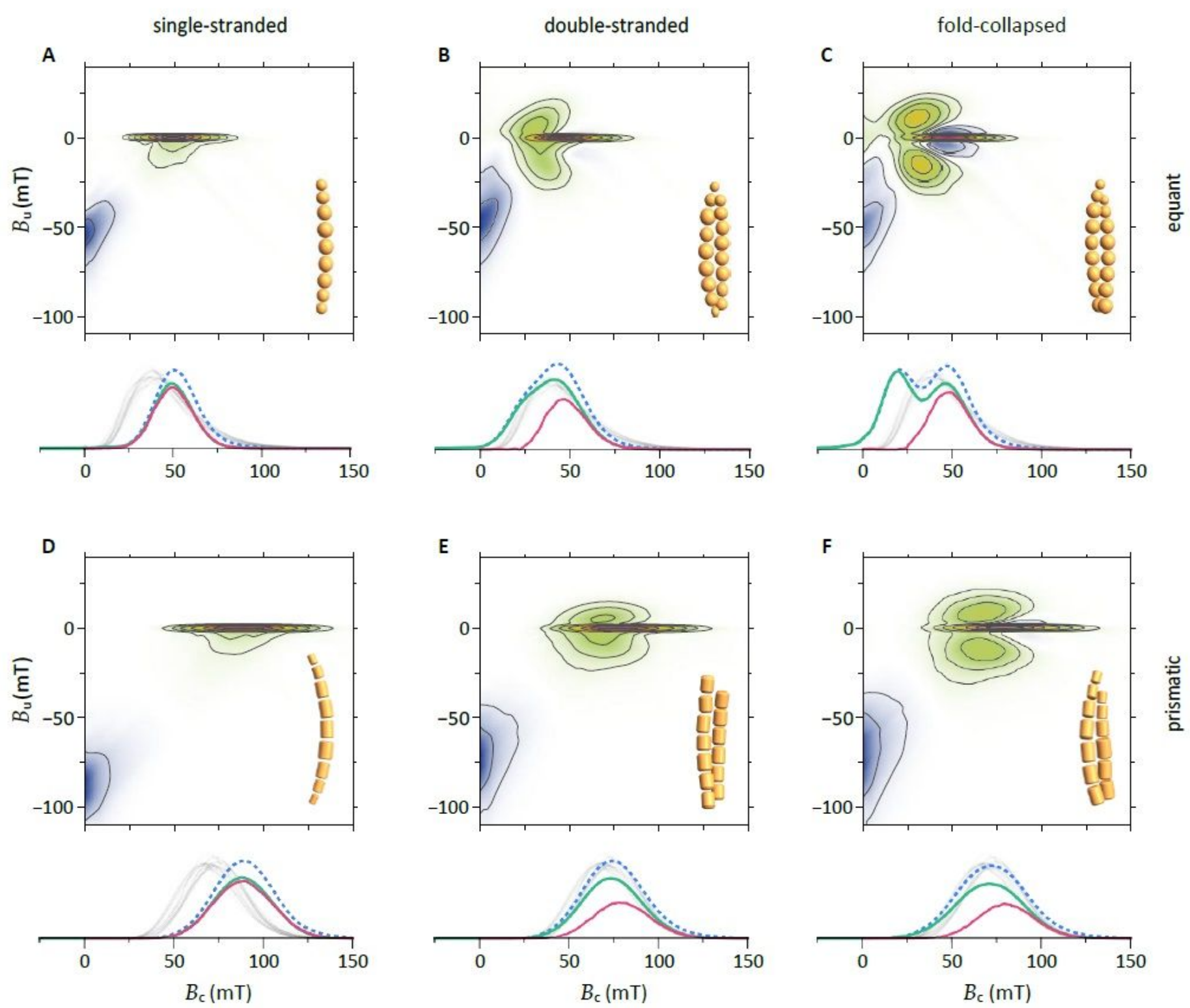

Figure 3

Electron microscopy images of wild-type and mutant AMB-1 cells. 

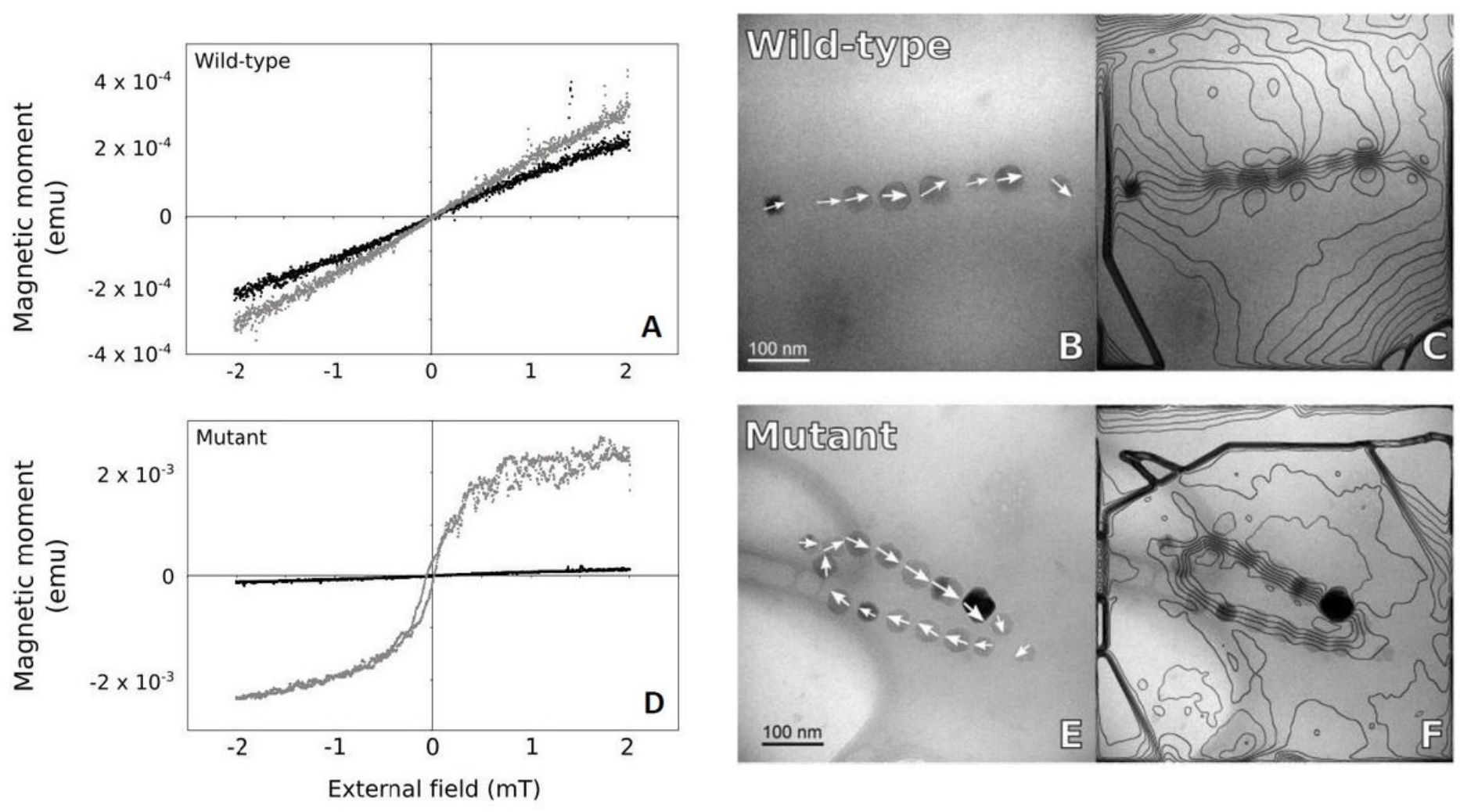

\section{Figure 4}

Native magnetic states of wild-type AMB-1 and the AMB-1 mutant. Magnetization of a suspension of (A) wild-type and (D) mutant AMB-1 cells in small fields varying between $\pm 2 \mathrm{mT}$, before (black) and after (gray) saturation in a $200 \mathrm{mT}$ field. Electron microscopy and corresponding magnetic phase contours determined by off-axis electron holography images of (B-C) wild-type and (E-F) mutant magnetite chains. 
A
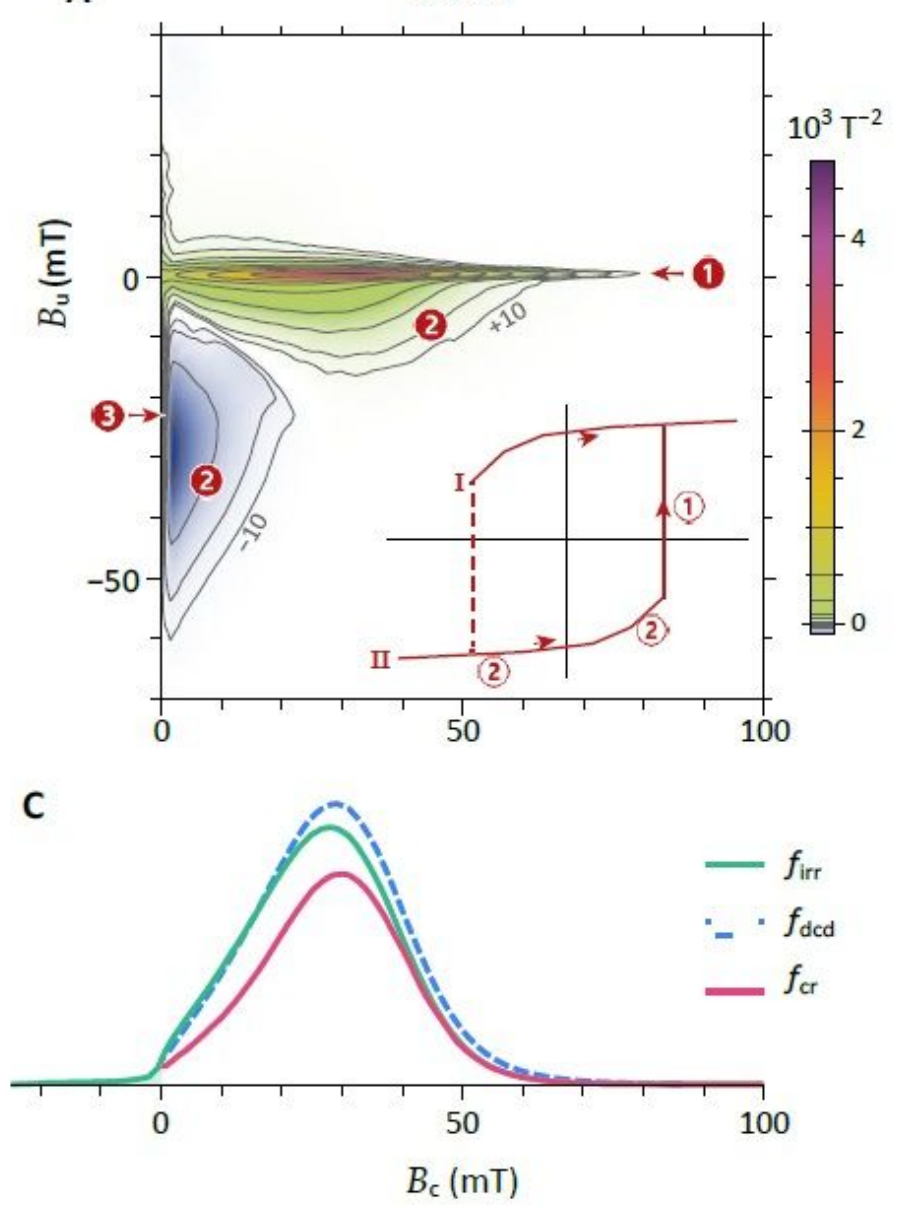

B
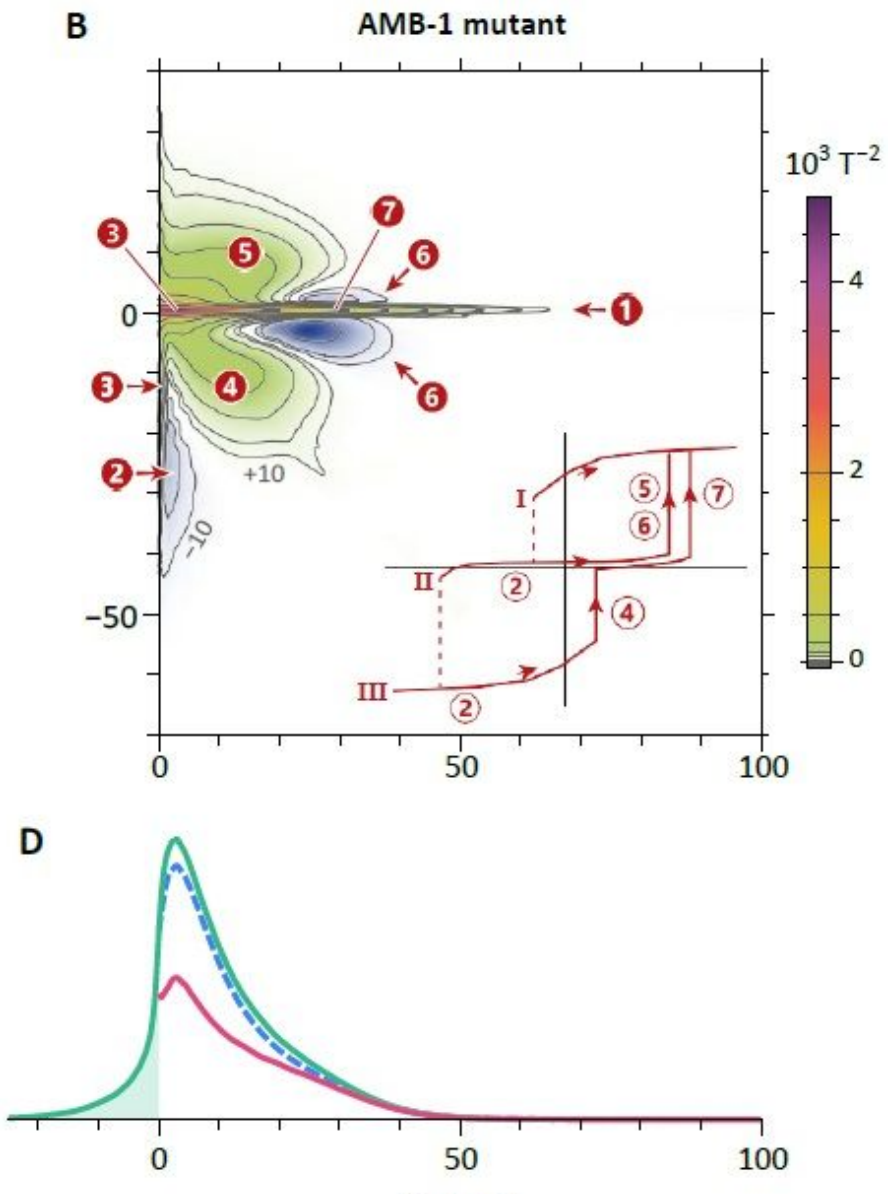

$B_{\mathrm{c}}(\mathrm{mT})$

\section{Figure 5}

Magnetic properties of wild-type AMB-1 and the AMB-1 mutant. (A-B) FORC diagrams normalized by the saturation magnetization, with same logarithmically spaced contours. Insets are schematic representations of the magnetization of an individual cell as a function of the applied field. Numbers highlight the following features: 1 - central ridge created by the magnetization jump through which the lowest curve merges with curve l; 2 - positive and negative contributions from reversible magnetic moment rotation (difference between the slopes of consecutive curves); 3 - signatures of magnetically viscous particles (vertical ridge and central ridge extending to the origin); 4 - nucleation of a negative FC state from a negative SD state (contribution of the first magnetization jump in curve III to the difference between curves II and III); 5 - annihilation of a positive FC state (contribution of the magnetization jump in curve II to the difference between curves I and II); 6 - same as 5, but for the difference between curve II and III; 7 - annihilation of a negative FC state (contribution of the second magnetization jump in curve III to the difference between curves II and III). (C-D) Coercivity distributions of wild-type AMB-1 and the AMB1 mutant, obtained from subsets of FORC data: firr - irreversible component of the ascending hysteresis branch, fdcd - DC demagnetization of Mrs, fcr - central ridge. The shaded area represents the firr contribution of SD magnetic states that cannot exist in a null field. 

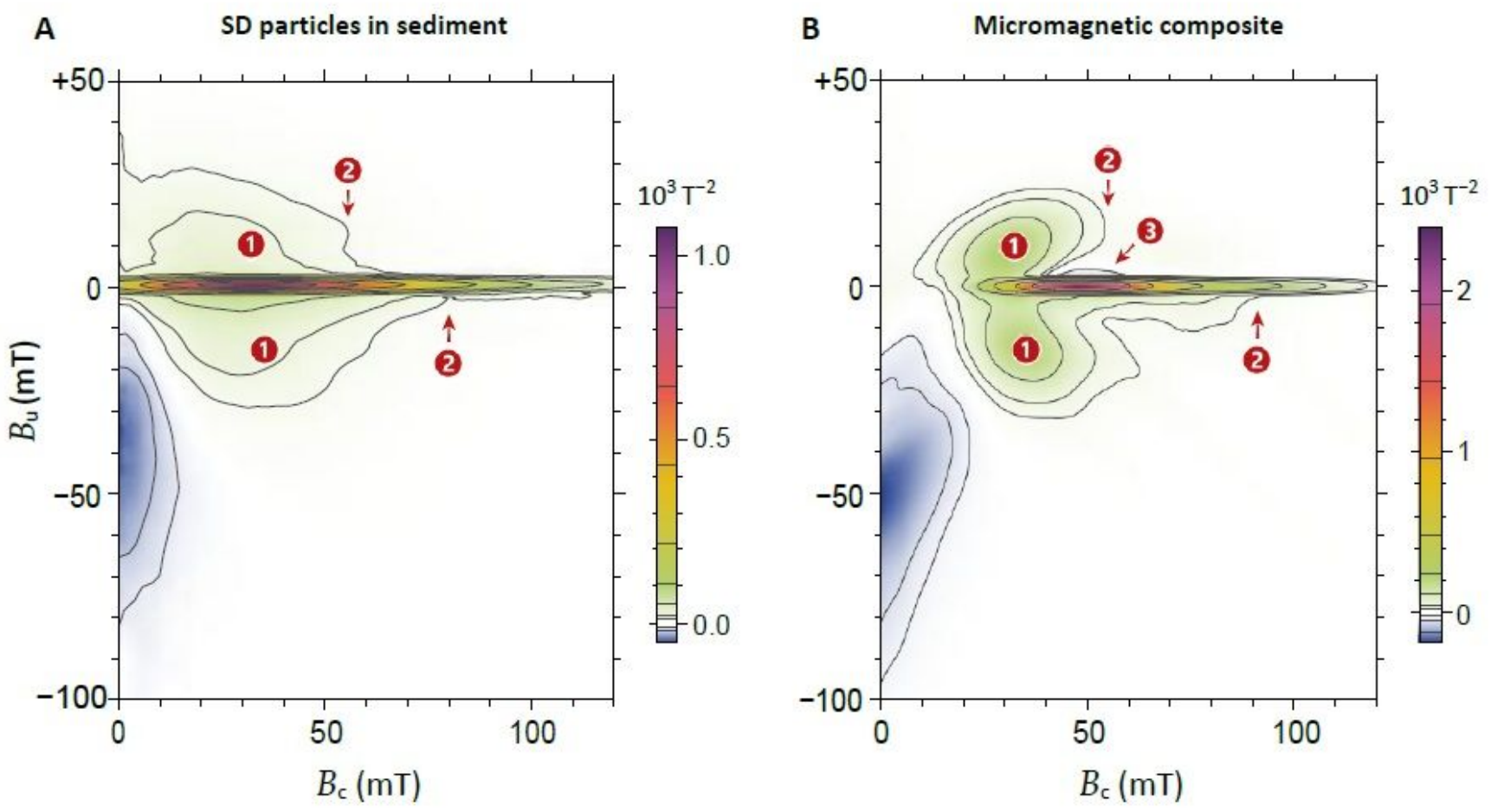

Figure 6

Comparison between the FORC signature of SD particles in a magnetofossil-rich sediment and synthetic results. (a) FORC diagram of in-situ $<1 \mu \mathrm{m}$ magnetite particles (mostly SD) in a pelagic sediment [data from ref. 20]. (b) FORC diagram of a synthetic composite obtained from the six micromagnetically modelled chain structures, with the following relative contributions to the saturation magnetization, chosen for a visual match with (a): $21 \%$ (single-stranded chains of equant magnetosomes), $4 \%$ (singlestranded chains of prismatic magnetosomes), $42 \%$ (native double-stranded chains of equant magnetosomes), $8 \%$ (native double-stranded chains of prismatic magnetosomes), $21 \%$ (fold-collapsed chains of equant magnetosomes), $4 \%$ (fold-collapsed chains of prismatic magnetosomes). The same field range and contour levels with respect to the maximum amplitude are used in both plots to ease the comparison. Numbers highlight the following features: 1 - nucleation/annihilation of FC states, 2 - highfield limit of FC state nucleation/annihilation (asymmetric about the central ridge), 3 - residual negative amplitude from the annihilation of positive FC states, uncompensated by positive amplitudes from intermediate coercivity contributions.

\section{Supplementary Files}

This is a list of supplementary files associated with this preprint. Click to download.

- SIAppendix1.pdf 